\title{
1 Association of milk microbiome in bovine clinical mastitis and their functional
}

\section{implications in cows in Bangladesh}

3 M. Nazmul Hoque ${ }^{1}$, Arif Istiaq ${ }^{1, \pm}$, Rebecca A. Clement ${ }^{2}$, Munawar Sultana ${ }^{1}$, Keith A. Crandall ${ }^{2}$,

4 AMAM Zonaed Siddiki ${ }^{3}$, M. Anwar Hossain ${ }^{1, \pm * *}$

$5{ }^{1}$ The Laboratory of Microbial Genetics and Bioinformatics, University of Dhaka, Dhaka 1000,

6 Bangladesh

$7 \quad{ }^{2}$ Computational Biology Institute, Milken Institute School of Public Health, the George Washington

8 University, USA

$9{ }^{3}$ Department of Pathology and Parasitology, Chittagong Veterinary and Animal Sciences University,

10 Chittagong-4202, Bangladesh

$11{ }^{ \pm}$Current status: PhD Student, Department of Developmental Neurobiology, Graduate School of Medical

12 Sciences, Kumamoto University, Japan

$13{ }^{ \pm}$Current status: Vice-Chancellor, Jashore University of Science and Technology, Jashore 7408,

14 Bangladesh

15

16

17

18

19

20

21

22

23 


\section{Abstract}

27 Milk microbiomes impose a significant influence on the pathophysiology of bovine mastitis. To

28 assess the association, we compared the microbiome of clinical mastitis (CM) and healthy $(\mathrm{H})$

29 milk samples through whole metagenomic deep sequencing. A total of 483.38 million reads

30 generated from both metagenomes were analyzed through PathoScope and MG-RAST, and

31 mapped to 380 bacterial, 56 archaeal, and 39 viral genomes. We observed distinct shifts and

32 differences in abundance between the microbiome of $\mathrm{CM}$ and $\mathrm{H}$ milk in phyla Proteobacteria,

33 Bacteroidetes, Firmicutes and Actinobacteria with an inclusion of $68.04 \%$ unreported and/or

34 opportunistic species in CM milk. Additionally, 14 archaeal and 14 viral genera were found to be

35 solely associated with CM. The functional metagenomics identified several pathways related to

36 bacterial proliferation and colonization such as metabolism, chemotaxis and invasion, immune-

37 diseases, oxidative stress, regulation and cell signaling, phage and prophases, antibiotic and

38 heavy metal resistance to be associated with CM. Therefore, the present study provides

39 conclusive data on milk microbiome diversity associated with bovine CM and its role in udder

40 health.

44 Keywords: Bovine Clinical and Healthy milk, Microbiome, Whole metagenome sequencing

45 (WMS), Taxonomic and Functional diversity. 


\section{Introduction}

51 Mastitis is one of the most prevalent diseases in the dairy industry with the highest clinical and

52 economic significance worldwide ${ }^{1}$. The condition usually happens when pathogenic microbes

53 enter the germ-free environment of the mammary gland, mostly by the disruption of the physical

54 barriers of the mammary quarters, requiring prompt and appropriate host defenses to prevent

55 colonization and subsequent disease pathology $y^{2}$. Diverse groups of microbes are known to

56 colonize the mammary quarters of cows, and have evolved novel mechanisms that facilitate their

57 proliferation, leading to clinical mastitis (CM). Despite knowledge of a few of these invading

58 microbial groups, the etiology of bovine mastitis is continuously changing, with new microbial

59 species identified as causing disease frequently. Additionally, although bacteria are the main

60 cause of mastitis ${ }^{3}$, other microbes like archaea, viruses and fungi might be associated with the

61 disease process ${ }^{4}$ and should therefore be investigated as well. During the progression of the

62 mastitis, dysbiosis of the milk microbiome can occur with the increase of opportunistic

63 pathogenic bacteria and reduction of healthy commensal bacteria ${ }^{5}$. Until recently, investigations

64 of microbiome associated with bovine mastitis have been mostly restricted to individual

65 pathogen isolation and characterization.

66 The disease is caused by epidemiologically diverse groups of microorganisms and categorized

67 into contagious and environmental mastitis ${ }^{6}$. The udder of the dairy cows is the primary reservoir

68 of contagious pathogens including Staphylococcus aureus, Streptococcus agalactiae,

69 Streptococcus dysgalactiae, Mycoplasma spp. and Corynebacterium bovis ${ }^{1,6}$. The involvement of

70 the bovine mammary gland microbiota in the host-pathogen interaction has little investigated 
71 except during the infectious episode ${ }^{7}$. Environmental pathogens such as Escherichia coli,

72 Klebsiella pneumoniae, Klebsiella oxytoca, Enterobacter aerogenes, Streptococcus dysgalactiae

73 and Streptococcus uberis ${ }^{1,6}$ can also be implicated in disease. Rapid advances in high-throughput

74 NGS technology and bioinformatics tools ${ }^{8}$ during the last decade have initiated a transition from

75 clinical microbiology to genomic characterization of the microbiome associated with infection,

76 including mastitis in lactating women ${ }^{5}$ and animals ${ }^{9}$. Shotgun whole metagenome sequencing

77 (WMS) produces a metagenome reflecting the total microbial makeup of a sample (pathogenic,

78 environmental, bacterial, fungal, viral) and has been used successfully to gain insights into the

79 phylogenetic composition and species diversity of a variety of microbiomes ${ }^{10}$, including profiling

80 of their functional attributes ${ }^{11}$. Thus, data can be generated regarding the identity and abundance

81 of genes related to microbial metabolism, virulence and antibiotic resistance enabling

82 identification of unknown etiological agents that play a role in mammary gland pathogenesis.

83 Overexpression of putative genes encoding immune suppression ${ }^{12}$, systemic oxidative stress $^{3}$,

84 and inflammatory processes ${ }^{13}$ are the crucial factors affecting the progression of CM.

85 Indiscriminate and overuse of antibiotics to treat mastitis is main cause of multidrug resistant

86 bacteria $^{14}$. Therefore, summarizing the variation in biota and protein functional diversity in

87 clinical and healthy milk microbiomes using cutting-edge genomic technologies like WMS ${ }^{15}$ and

88 associated bioinformatic tools is essential to understanding the pathophysiological conditions of

89 bovine CM. Here we report the first study of its kind where high-throughput sequencing data (on

90 an average 23.01 million reads per sample) were generated to investigate the microbiome of

91 bovine $\mathrm{CM}$ and $\mathrm{H}$ milk ${ }^{16}$. The results revealed that cows suffering from $\mathrm{CM}$ milk had a distinct

92 microbial community with reduced diversity, higher relative abundance of opportunistic

93 pathogens, and altered protein functions compared to their healthy counterparts. 


\section{Results}

97 Structure and composition of the bovine milk microbiome. Compared to healthy $(\mathrm{H})$

98 microbiomes, clinical mastitis (CM) milk microbiomes showed significantly reduced Shannon-

99 estimated microbial richness $(\mathrm{H} ; p=0.005, \mathrm{CM} ; p=0.007, \mathrm{U}$ test $)$. Species richness in both

100 metagenomes also differed significantly between two bioinformatics tools (PS; $p=0.039$, MR;

$101 p=0.001$, U test) (Supplementary Fig. 1). Beta diversity (PCoA) revealed significant microbial

102 disparity ( $p=0.001$ ) between CM and H sample groups (Supplementary Fig. 2). At phylum level,

103 NMDS showed distinct diversity differences between the sample categories (Supplementary Fig.

$1043)$.

105 At the domain level, bacteria were the most abundant community, with an average abundance of

$10698.00 \%$, followed by eukaryotes $(1.80 \%)$, archaea $(0.02 \%)$, viruses $(0.04 \%)$, and unassigned

107 sequences $(0.002 \%)$ (Supplementary Data 1$)$. Though the relative abundance of microbes was

108 higher in CM compared to $\mathrm{H}$ milk, the abundance fluctuated more (CV=886.90 vs 511.80; PS,

$109 \mathrm{CV}=1521.41$ vs $1221.92 ; \mathrm{MR})$. The unique and shared distribution of microbial taxa found in

$110 \mathrm{CM}$ and $\mathrm{H}$ samples by two analytic tools is represented in Venn diagrams (Fig.1). A total of 363

111 bacterial species in CM, and 146 species in $\mathrm{H}$ metagenomes were detected in PS analysis, of

112 which $116(29.51 \%)$ species shared in the both conditions (Fig. 1a). However, through MR

113 pipeline, 356 and 251 bacterial genera were detected in CM and $\mathrm{H}$ samples respectively, whereas

$114227(63.8 \%)$ genera were common in both metagenomes (Fig. 1b). By comparing the detected

115 bacterial genera between two analytic tools, 98 unique genera were identified, of them $62.24 \%$

116 genera were solely associated with the onset of bovine CM (Fig. 1c, Supplementary Data 2). In 
117 addition, MR detected 54 and 42 archaeal, and 35 and 25 viral genera, respectively in CM and $\mathrm{H}$

118 samples, and among them $25.00 \%$ and $35.00 \%$ archaeal and viral genera respectively had sole

119 association with CM (Fig. 1d, e). Unlike MR, PS detected only one archaeal genera

120 (Methanobrevibacter) in CM and none in $\mathrm{H}$ samples.

122 CM-associated bacteria changes at the genus level. The current microbiome study

123 demonstrated notable differences among the microbial community in $\mathrm{CM}$ and $\mathrm{H}$ milk in both

124 bioinformatics tools. Proteobacteria, Bacteroidetes, Firmicutes and Actinobacteria (contributing

125 to $96.51 \%$ of the total sequences, $\mathrm{U}$ test, $p=0.001$ ) were the four most abundant phyla in PS and

126 MR analyses. The relative abundance of the top 40 bacterial genera was compared between CM

127 and $\mathrm{H}$ cohort through PS (Fig. 2) analyses. Among the predominating phyla, Proteobacteria was

128 among the most diverse, and included a wide variety of genera including Acinetobacter,

129 Pseudomonas, Escherichia, Vibrio, Erwinia, Pantoea. The phylum Firmicutes was dominated by

130 Streptococcus, Enterococcus, Staphylococcus, and Bacillus genera while Chryseobacterium,

131 Porphyromonas and Prevotella genera were predominating in Bacteroidetes phylum, and

132 Corynebacterium was the most abundant genus in phylum Actinobacteria. Among the detected

133 genera either of the tool, Acinetobacter (60.14\%), Campylobacter (10.93\%), Pantoea (0.66\%),

134 Klebsiella (0.63\%), Kluyvera (0.42\%), Salmonella (0.31\%), Enterobacter (0.30\%), Shewanella

135 (0.30\%), Escherichia (0.28\%), Citrobacter (0.15\%) and Bacillus (0.10\%) had higher mean

136 relative abundance in CM samples in both analytic tools, while rest of the genera had relatively

137 lower mean abundance $(<0.10 \%)$. In contrast, the $\mathrm{H}$ milk metagenomes also had higher mean

138 relative abundance of genus Acinetobacter (52.90\%) in PS and MR followed by Pseudomonas

139 (22.81\%), Micromonospora (10.57\%), Eubacterium (5.37\%), Catenibacterium (2.12\%) and 
140 Ralstonia (0.12\%)genera, and rest of the genera had much lower abundance $(<0.10 \%)$. In

141 general, MR detected higher number of microbial genera than PS (Supplementary Tables 2\&3),

142 however results from the both tools were concordant, with $98.00 \%$ of the total microbial

143 abundance composed of shared genera (Supplementary Table 4, Supplementary Data 2).

144 CM-associated bacteria changes at the strain level. We further investigated whether strain

145 level relative abundances of the bacteria differed between CM and $\mathrm{H}$ samples (Fig. 3, 4). The

146 CM milk metagenome had significantly $(p=0.001)$ higher number of bacterial species than the

$147 \mathrm{H}$ milk, and among the detected species $62.85 \%$ had unique association with bovine CM, and

$1487.63 \%$ were solely found in $\mathrm{H}$ milk (Fig. 1a). The presence of few predominating bacterial

149 species in both categories of samples suggests that the crucial differences might be occurring at

150 the strain level, and most of the species identified in each sample were represented by a single

151 strain. The CM milk metagenome was dominated by 26 strains (7.16\%) of Acinetobacter

152 species while Pseudomonas, Streptococcus, Corynebacterium, Staphylococcus, Enterococcus,

153 Bacillus, and Escherichia species were represented respectively by 22, 16, 12, 11, 8, 7 and 6

154 different strains. However, in both metagenomes, Acinetobacter johnsonii XBB1 remained as the

155 most abundant strain with a relative abundance of $39.03 \%$ and $31.23 \%$ respectively in $\mathrm{CM}$ and $\mathrm{H}$

156 samples. The other predominant strains in CM metagenome were Campylobacter mucosalis,

157 Bacillus mycoides, Klebsiella pneumoniae subsp. pneumoniae HS11286, Leclercia

158 adecarboxylata, Escherichia coli str. K-12 substr. MG1655, Escherichia coli O157:H7 str.

159 Sakai, Escherichia coli UMN026, Escherichia coli IAI39, Staphylococ cusaureus subsp. aureus

160 NCTC 8325, Staphylococcus xylosus, Bacillus subtilis subsp. subtilis str. 168, Mycobacterium

161 sp. Root 265, Macrococcus caseolyticus. Importantly, this study demonstrated that $68.04 \%$ of the

162 detected bacterial strains were exclusively found in CM milk metagenome, and among them 
Pantoea dispersa EGD-AAK13, Klebsiella oxytoca, Kluyvera intermedia, Shewanella

164 oneidensis MR-1, Kluyvera ascorbata ATCC 33433, Klebsiella aerogenes KCTC 2190,

165 Kluyvera cryocrescens NBRC 102467, Acinetobacter pittii PHEA-2, Pseudomonas mendocina

166 ymp, Acinetobacter gyllenbergii NIPH 230 were the most predominant strains. Furthermore,

167 most of these strains were previously unreported and possibly played an opportunistic role in the

168 mammary gland pathogenesis (Supplementary Data 2, Supplementary Table 5).

169

170 CM-associated changes of archaea and viruses at the genus level. Archaea and viruses were

171 detected in the samples of the both metagenomic groups; $\mathrm{CM}$ and $\mathrm{H}$ milk. The $\mathrm{CM}$ metagenome

172 was dominated by Methanosarcina (41.94\%), Methanococcoides (19.58\%), Methanococcus

173 (12.30\%), Methanocaldococcus (2.59\%), Methanobrevibacter (1.85\%), Thermococcus (1.79\%),

174 and Methanosphaera $(1.53 \%)$ archaeal genera with a lower relative abundance $(<0.05 \%)$ of the

175 rest of the genera (Fig. 5 a, Supplementary Data 2). Interestingly, none of the archaeal genus was

176 detected in one CM sample (Ctg3C2). In contrast, Methanoplanus (14.69\%), Methanoculleus

177 (12.85\%), Euryarchaeota (4.67\%), and Haloarcula (1.50\%) were the most abundant archaeal

178 genera in $\mathrm{H}$ samples. The viral fraction of the current bovine milk microbiome was largely

179 dominated by the members of the Caudovirales order, represented by the Podoviridae,

180 Siphoviridae, and Myoviridae families. The predominating viral genera found in CM were

181 Epsilon15-like viruses (15.78\%), P2-like viruses (10.12\%), Myovirus (8.18\%), Lambda-like

182 viruses (8.06\%), Bpp-1-like viruses (7.12\%), phiKZ-like viruses (4.35\%), Betaretrovirus

183 (2.01\%), Pl-like viruses (1.79\%) and T4-like viruses (1.79\%). The $\mathrm{H}$ milk however had

184 relatively higher abundance of Siphovirus (55.85\%), Podovirus (12.49\%), T1-like viruses

185 (3.44\%) and P22-like viruses (1.71\%) (Fig. 5 b, Supplementary Data 2). 
187 Microbial metabolic functions associated with CM. MR simultaneously analyzed and

188 compared the taxonomic compositions and functional profile in our metagenomic sequences in

189 several ways. On average, the putative genes with known predicted protein and known functions

190 were $3.94 \%$ and $5.51 \%$, respectively suggesting that a large proportion of the genes encoding for

191 different functional properties are yet unknown (Supplementary Data 1). By comparing the

192 number of genes assigned to each KEGG pathway between the groups, we found a series of

193 significant differences $(p=0.001)$ that lead to the functional divergence among $\mathrm{CM}$ and $\mathrm{H}$ milk

194 microbiotas. The PCoA analysis at level 3 subsystems showed that CM metagenome separately

195 distributed from $\mathrm{H}$ milk metagenome indicating significant functional differences $(p=0.035)$

196 (Supplementary Fig. 4). In the comparative analysis, we found that genes associated with

197 metabolism (central carbohydrate, amino acids, cofactors, vitamins, prosthetic groups and

198 pigment), substrate dependence, clustering-based subsystems, cell motility (bacterial

199 chemotaxis, flagellar assembly, invasion of epithelial cells), phases, prophages, transposable

200 elements and plasmids, regulation and cell signaling, stress response, virulence, disease and

201 defense, immune and infectious diseases, cancer pathways were significantly $(p<0.05)$ over

202 represented and positively correlated with bovine CM (Fig. 6, 7, Supplementary Data 3).

203 Genes associated with citrate synthase (CS, gltA), fumarate hydratase class I (fumA, fumB),

204 oxidative phosphorylation, bacterial translation, ribosome biogenesis and tRNA amino-

205 acylation were significantly enriched in the metabolic pathways of CM associated

206 microbiomes. The $\mathrm{CM}$ associated microbiotas had significantly $(p<0.001)$ higher relative

207 abundance $(50.51 \%)$ of genes coding for benzoate degradation than the $\mathrm{H}$ milk biomes

208 (36.41\%). The CM milk microbes had upregulation of genes for energy metabolism including 
209 one carbon metabolism, sulfur and methane metabolism than $\mathrm{H}$ milk microorganisms. The

210 relative abundance of genes encoding $\mathrm{ABC}$ transporter (38.97\%) and bacterial chemotaxis

$211(68.61 \%)$ remained significantly higher in CM microbes than those detected in H milk biomes

$212(p<0.005)$. Among the pathways in infectious diseases, genes coding for epithelial cell signaling,

213 epithelial cells invasion, Legionellosis, Vibrio cholerae pathogenic cycle, Staphylococcus

214 aureus, Salmonella and pathogenic Escherichia coli infection were mostly abundant in CM

215 metagenome. Likewise, there was a predominant abundance of genes responsible for glutathione

216 S-transferase (GST), breakpoint cluster region protein (BCR1), fumarate hydratase class II

217 (fumC), and pyruvate kinase (pk) in different pathways causing mammary gland cancer. We

218 observed significantly higher abundance of genes encoding apoptosis in CM microbiomes, and

219 in contrast, the relative abundance of proteins that are involved in various cellular functions (cell

220 growth and differentiation) through the p53 signaling pathway remained higher in $\mathrm{H}$ milk

$221(\mathrm{p}<0.05)$. The CM milk microbiomes had significantly $(p<0.001)$ higher number of reads

222 (64.29\%) coding for severely combined immune deficient gene adenosine deaminase (ADA)

223 than $\mathrm{H}$ milk microbes (28.58\%) (Supplementary Fig. 5). Furthermore, sporulation related

224 hypotheticals and CRISPR-associated proteins (Cas1, Cas2, and Cas3) remained higher in CM

225 metagenomes than H milk microbes (Supplementary Data 3).

226 We found that the CM microbiotas had significantly higher abundance of genes encoding for

227 oxidative stress (36.46\%), pathogenicity islands (10.13\%), phage related transposable elements

228 (19.48\%), phage packaging machinery (6.37\%), phage replication (6.70\%) and phage

229 regulatory gene expression (7.10\%) than those of $\mathrm{H}$ milk biomes $(p<0.003)$. However, the

230 phage lysogenic conversion related genes remained higher in abundance among the healthy

231 milk microbes. A deeper look at microbial genes associated with regulation and cell signaling 
232 revealed that $\mathrm{CM}$ microbes had significantly higher expression of this gene compared to

233 healthy milk microbiotas $(p=0.001)$. Within this subsystem, genes coding for two-component

234 regulatory system BarA-UvrY (SirA; CM= 85.78\% vs H=67.41\%), pericellular trafficking and

235 cell invasion- the membrane type-1 matrix metalloproteinase (MT1-MMP; CM= 86.59\% vs

$236 \mathrm{H}=73.80 \%)$, programmed cell death $(\mathrm{CM}=55.00 \%$ vs $\mathrm{H}=28.57 \%)$, and intra-membrane

237 regulatory proteolytic pathway- endoplasmic reticulum chaperon $\operatorname{grp} 78(\mathrm{BiP} ; \mathrm{CM}=92.85 \%$ vs

$238 \mathrm{H}=71.42 \%$ ) were predominantly found to be associated with the onset of bovine CM. We also

239 identified novel associations of biofilm formation (BF) properties among the microbes

240 identified in both metagenomes. The relative abundance of genes coding for protein $Y j g \mathrm{~K}$ cluster

241 linked to biofilm formation, biofilm PGA synthesis, deacetylase PgaB, N-glycosyltransferase

$242 P g a \mathrm{C}$, and auxiliary protein $P g a \mathrm{D}$ had statistically over expression among mastitis causing

243 pathogens $(p=0.035)$. In contrast, the genes coding for quorum sensing (QS) in particular to QS

244 in Yersinia, Pseudomonas and Vibrio remained over expressed in H milk metagenomes.

245 Moreover, of the assigned reads to different levels SEED subsystems (6.45 million), 2.63\%

246 mapped against 30 and 28 different resistance to antibiotic and toxic compounds (RATC) genes

247 respectively in $\mathrm{CM}$ and $\mathrm{H}$ milk metagenomes (Fig. 8, Supplementary Data 3). Among them,

248 genes encoding multidrug resistance to efflux pumps, cluster ( $m d t \mathrm{ABCD})$, operon $(\mathrm{Cme} \mathrm{ABC})$

249 and MAR locus, methicillin resistance in Staphylococci, vancomycin resistance, arsenic and

250 chromium compounds resistance had two-fold higher relative abundances in CM microbiotas

251 than $\mathrm{H}$ milk biomes. There was 5 to 7-fold over expression of multidrug resistance to MAR locus

252 and mercury resistance genes in $\mathrm{CM}$ microbes than $\mathrm{H}$ milk organisms. In addition, $\mathrm{CM}$ causing

253 microorganisms harbored two additional resistance genes; multidrug resistance to operon

254 (mdtRP) and aminoglycoside adenyltransferase (Supplementary Data 3). 


\section{Discussion}

258 During the last decade metagenomics has helped to shed some light onto the "known unknown",

259 component of the milk microbiome and to enable insights into its taxonomic composition,

260 dynamics, and importance to cows udder health homeostasis. Metagenomic deep sequencing

261 (WMS) of bovine milk has uncovered previously overlooked microbial populations of high

262 complexity with potential roles in regulation of overall microbiome composition and their

263 functional attributes, and in the onset, progression, and treatment strategies of bovine CM. Yet

264 today, 16SrRNA gene sequencing remained as the key approach for studying milk microbiomes,

265 and findings are mostly limited to bacterial identification at the genus level ${ }^{5,9,17}$, though this

266 method has serious inherent limitations ${ }^{18}$. However, little is known about the association of other

267 microbes (archaea and viruses), microbiome shift, and particular functional changes during the

268 progression of the disease. The noteworthy findings of the present WMS study are the taxonomic

269 profiling of bacteria at both the species and/or strain-level, the possible association of the

270 archaeal and viral fractions with bacterial mastitis, and the crosstalk between the identified

271 microbiomes and their functional genomics in the association of bovine CM.

272 The findings generated by PS and MR are much higher in taxonomic resolution and predicted

273 proteins functions, and are consistent with previous 16 SrRNA gene based studies ${ }^{1,9,17}$. The core

274 bacteria associated with bovine CM such Acinetobacter, Pseudomonas, Klebsiella, Escherichia,

275 Enterobacter, Staphylococcus, Streptococcus, Bacillus, Pantoea, Shewanella, Ralstonia etc.

276 remained consistent in both analytic tools although their relative abundances varied even within 
277 same sample group. The study demonstrated that in spite of having relatively higher taxonomic

278 abundance, the $\mathrm{CM}$ associated microbiomes fluctuate more within the samples than those

279 identified in $\mathrm{H}$ milk metagenome corroborating several recent findings ${ }^{5,17,18,19}$. To date, around

28050 bacterial genera have been reported in bovine milk through 16SrRNA-based

281 metagenomics ${ }^{1,9,17,20}$, while our current WMS study detected 356 and 251 bacterial genera in CM

282 and $\mathrm{H}$ milk, respectively indicating the increased discriminatory power of this cutting-edge

283 technology in identifying microbiomes ${ }^{10,15}$. The observed increase in phylum-level signature of

284 Proteobacteria, Bacteroidetes, Firmicutes and Actinobacteria in CM milk independent of quarter,

285 parity, and breeds of the cows is almost consistent with many of the previous studies ${ }^{5,9,17,26}$.

286 Furthermore, the CM milk metagenome had an inclusion of $68.04 \%$ previously unreported

287 bacterial species, most of which are opportunistic in nature. Before now, no substantial

288 information is available regarding the association of different strains of Acinetobacter with

289 bovine mastitis, which are opportunistic pathogen to causing CM by acquiring exogenous DNA

290 from other bacteria through horizontal gene transfer, and concomitantly becomes a reservoir of

291 resistant genes ${ }^{23}$. In a recent study, highest association of Acinetobacter causing bubaline $\mathrm{CM}^{7}$

292 has been reported supporting our present findings. The $\mathrm{H}$ milk metagenome had higher relative

293 abundance of soil or environment (Micromonospora) and animal skin (Pseudomonas) associated

294 bacteria, which can act as potential opportunist by attacking weak or injured tissues of teats or

295 mammary glands ${ }^{25,26}$, and can cause acute and/or chronic mastitis ${ }^{27}$. Furthermore, the

296 predominantly identified CM associated bacteria, Klebsiella pneumoniae is an opportunistic

297 environmental pathogen, and transmission of this bacterium might occur from contaminated

298 feces and bedding materials ${ }^{28}$ to help in causing CM in healthy mammary glands and/or quarters.

299 Gut microbiome plays a key role in maintenance of nutrition, host defense and immune 
300 development $^{29}$, and we revealed a close association between gut microbiota and milk microbes in

301 the pathogenesis of bovine $\mathrm{CM}^{30}$. Additional support for this finding includes, the potential

302 existence of an endogenous entero-mammary pathway, through which gut bacteria migrate to the

303 mammary gland, and this could explain the predominating presence of gut bacteria such as phyla

304 Proteobacteria, Bacteroidetes, Firmicutes, Actinobacteria, Fusobacteria, and Tenericutes, with

305 Acinetobacter, Campylobacter, Bacillus, Enterobacter, Staphylococcus, Streptococcus, Kocuria

306 genera in $\mathrm{CM}$ milk $^{30-32}$. These pathogens use very efficient strategies to evade host defenses in

307 order to colonize and invade mammary tissues through adhesion ${ }^{33}$, thereby damage host cells and

308 fight with cows immune systems to producing clinical and/or chronic mastitis ${ }^{33-35}$. Despite

309 relatively lower abundance, the rest of the bacterial genera, species/strains detected across the

310 clinical milk metagenome had symbiotic network, and positive correlation with CM. Our study

311 marks an additional step towards identifying the significant co-occurrence of archaea and viruses

312 with bacterial population in bovine milk. Unlike bacteria, the relative abundance and diversity of

313 archaea $^{36}$ and viruses ${ }^{37}$ remained substantially lower. Currently there is no extensive evidence

314 supporting the role of archaea and viruses in the pathogenesis of bovine mastitis, however these

315 microbes mostly cease the opportunity during the pathophysiological changes in the mammary

316 glands created by bacteria ${ }^{38}$. The full spectrum of outcomes from these archaea-cows

317 interactions, whether it altered host mammary gland physiology remained as a mystery. Thus, it

318 is hypothesized that archaea might follow the exact mechanisms of bacterial pathogens

319 producing bovine $\mathrm{CM}^{36}$. Most of the detected viral genera belonged to the order Caudovirales

320 which consists of the three families of tailed bacterial viruses (bacteriophages) infecting bacteria

321 and archaea. The host range of Caudovirales is very broad and includes all major bacterial phyla

322 found in both metagenomes: Firmicutes, Bacteroidetes, Proteobacteria, and Actinobacteria. This 
323 corresponded with an increase relative abundance of these bacterial taxa in CM milk samples

324 together with an over presentation of Caudovirales taxa compared with H milk metagenome ${ }^{39}$. In

325 addition, we revealed the association of Herpesvirales (Macavirus and Rhadinovirus genera)

326 with bovine $\mathrm{CM}^{39,40}$. Our current findings demonstrated that viruses neither cause bovine

327 mastitis directly nor play role in the initiation of the disease process, but later, when bacterial

328 infection of the udder occurs, they replicate in the immune and epithelial cells of the udder

329 and/or milk ducts, and may act as predisposing factor as well as primary etiological agent for

330 more severe and prolonged mastitis ${ }^{41}$.

331 The KEGG pathways and SEED subsystems of MR pipeline uncovered significant differences

332 in microbial metabolic functions in both metagenomes ${ }^{5,42}$ as supported by several previous

333 reports on mastitis in lactating $\operatorname{cows}^{9}$ and women ${ }^{5}$. The CM microbiota had significantly higher

334 abundance of Proteobacteria and Bacteroidetes, which are well-known bacteria to utilizing

335 milk oligosaccharides; one carbon metabolism ${ }^{43}$. Genes associated TCA cycle (gltA, fumA)

336 and energy metabolism (oxidative phosphorylation) remained over expressed in CM

337 microbiomes, which might be associated with host-pathogen interactions during the

338 progression of bovine mastitis ${ }^{44,45}$. Increased benzoate degradation by different strains of

339 Acinetobacter and Klebsiella in CM metagenome through TCA cycle is thought to promote

340 bacterial growth and virulence factors expression during pathogenesis ${ }^{46,47}$. To elucidate the

341 role of bacterial chemotaxis in bovine mastitis, we found that genes coding for bacterial

342 chemotaxis is predominantly abundant in CM milk microbiomes suggesting their role in early

343 phase of mastitis for attachment to or entry into the udder tissues and virulence regulation ${ }^{48}$.

344 The p38 signaling pathway exerts its biological effects in the pathophysiology of bovine CM

345 through several complex biologic processes including expression of many cytokines, 
346 transcription factors, cell surface receptors, enzymes and oxidative stress mediators ${ }^{49}$. The p38

347 mediated transcriptional regulation of matrix metalloproteinase-2 (MMP-2 $)^{50}$ and pro-

348 inflammatory mediator cyclooxygenase-2 $(\mathrm{COX} 2)^{49}$ can potentially contribute to mammary

349 gland cancer and/or glandular mastitis. The up-regulation of genes coding for programmed-cell

350 death during host-pathogen interactions in $\mathrm{CM}$ is associated with increased secretion of

351 bacterial toxins, or pro-inflammatory mediators ${ }^{51}$. Diverse groups of microbiomes (bacteria

352 and viruses) causing bovine CM might induce cell death with their apoptotic features ${ }^{51}$. The

353 predominantly identified membrane type-1 matrix metalloproteinase (MT1-MMP) across the

$354 \mathrm{CM}$ metagenome is a pro-invasive protease regulating various cellular functions, macrophage

355 migration to the inflamed mammary tissues, and causes adenocarcinoma in cows udder ${ }^{52}$. We

356 demonstrated that endoplasmic reticulum chaperon (GRP78/BiP) associated gene signatures

357 are highly expressed in CM microbiotas, which can promote tumor proliferation and metastasis

358 in mammary tissues ${ }^{53}$. Biofilm formation can be a strain specific or genetically linked trait,

359 representing a selective advantage in pathogenesis of mastitis. The relatively over expression

360 of genes encoding protein YjgK cluster linked to biofilm formation, and biofilm PGA synthesis

361 in $\mathrm{CM}$ microbiomes is in accordance with several earlier reports ${ }^{54}$. Moreover, biofilm

362 formation can also be harmful to host tissues since they can promote the phagocyte release of

363 lysosomal enzymes, proliferation of reactive oxygen and nitrogen species, and transfer of

364 antibiotic resistance ${ }^{55}$. The observed increase abundance of genes for primary immune

365 diseases; adenosine deaminase (ADA) in CM pathogens is responsible for inhibition of $\mathrm{T}$ cell

366 maturation and lymphocytic proliferation ${ }^{56}$, very low CD4 count ${ }^{57}$, cell-to-cell

367 communication $^{58}$, and therefore could be used as a selective marker for bovine CM diagnosis.

368 CRISPR/Cas systems are present in both pathogenic and commensal organisms found in 
369 bovine milk, and play critical roles during the pathogenesis of mastitis by evading the hosts 370 defense system particularly under stress condition ${ }^{59}$. The type III and IV secretion systems

371 found on the pathogenicity islands of CM associated microbes are capable of producing

372 immunosuppression in cows by delivering effector proteins ${ }^{960}$. Phages, which are the regulators

373 of bacterial population, play important and diverse roles in all bacterial ecosystems ${ }^{61}$, but their

374 precise impact on the milk microbiota is far from being understood. The relatively over

375 presentation of genes coding for phage related transposable elements, phage packaging

376 machinery, phage replication and phage regulatory gene expression in $\mathrm{CM}$ microbes may

377 suggests that bacteriophages participate in the horizontal gene transfer among the members of

378 bovine milk microbiomes, and ultimately to mammary gland pathogens ${ }^{39}$. We propose that as

379 obligate parasites bacteriophages naturally found in raw milk, replicate in bacterial host, follow

380 the lysogenic cycle, disrupt host metabolism and, ultimately, causing death of bacterial cell

381 during the immunosuppression states of the cows, and finally release new phage particles ${ }^{39,40}$.

382 Bovine milk microbiomes are a wide source of resistance to antibiotic and toxic compounds

383 (RATC) genes and the pathogenic bacteria within this potential reservoir are becoming more

384 resistant. The current metagenomic deep sequencing provides a wealth of information not only

385 on RATC genes, but on the entire gene content thereby enabling the identification of the

386 community composition and metabolic profile. We found that all of the samples in both

387 metagenomes harbored RATC genes (2.63\%) indicating their wide and indiscriminate use in

388 Bangladeshi dairy farms. However, most of the resistant genes in RATC functional groups

389 remained predominantly higher in CM milk microbes. Although the knowledge on uncontrolled

390 spread of antibiotics resistant genes in bovine mastitis pathogens ${ }^{62}$ are increasing, but

391 information on heavy metal resistance is yet unavailable. This worrisome trend in increasing 
392 RATC against mastitis pathogens has become a major concern for the dairy holders of

393 Bangladesh, given the seriousness of such problems; effective therapies using alternative

394 medicines are needed for successful prevention and control of bovine mastitis.

395 The novel WMS technology in combination with improved bioinformatic analysis of milk

396 microbiome identifies the comparative microbial communities associated with bovine CM and

397 H quarters. The significant differences in the microbiome compositions and protein functional

398 diversities in two groups implicated the association in the progression of the

399 pathophysiological conditions of the disease.

400

401 Methods

402 Study population and sampling. Details of study population and collected samples are

403 presented in Supplementary Table 1. A total of 21 milk samples (14, CM and 7, H) from 21

404 lactating crossbred cows at their early stage of lactation (within 10-40 days of calving) were

405 collected from three districts of Bangladesh (Chattagram=12, Dhaka= 3, Gazipur=6). The

406 sampling patterns followed collection of two CM and one $\mathrm{H}$ milk samples from the same farm.

407 Approximately 15-20 ml of milk from each cow was collected in a sterile falcon tube during the

408 morning milking (8.0-10.0 am) with emphasis on pre-sampling disinfection of teat-ends and

409 hygiene during sampling ${ }^{1,63}$. The milk samples were then transported to the laboratory, and

410 stored at $-20^{\circ} \mathrm{C}$ until DNA extraction.

411 DNA extraction and sequencing. Genomic DNA (gDNA) was extracted by an automated DNA

412 extraction platform (Promega, UK) following previously described protocols ${ }^{5,16}$. DNA quantity

413 and purity was determined with NanoDrop (ThermoFisher, USA) by measuring 260/280

414 absorbance ratios. Sequencing libraries were prepared with Nextera XT DNA Library 
415 Preparation $\mathrm{Kit}^{64}$ according to the manufacturer's instructions, and paired-end $(2 \times 150 \mathrm{bp})$

416 sequencing was performed on a NextSeq 500 machine (Illumina Inc., USA) at the Genomics

417 Core facility at The George Washington University. Our metagenomic DNA yielded 483.38

418 million reads with an average of 23.01 million (maximum=35.10 million, minimum=6.77

419 million) reads per sample (Supplementary Data 1).

420 Sequence reads preprocessing. The resulting FASTQ files were concatenated and filtered

421 through $\mathrm{BBDuk}^{13}$ (with options $\mathrm{k}=21$, mink=6, $\mathrm{ktrim}=\mathrm{r}, \mathrm{ftm}=5$, qtrim=rl, trimq=20, minlen=30,

422 overwrite=true) to remove Illumina adapters, known Illumina artifacts and phiX. Any sequence

423 below these thresholds or reads containing more than one ' $\mathrm{N}$ ' were discarded. On an average,

42420.16 million reads per sample (maximum=32.33 million, minimum=4.71 million) passed

425 quality control step (Supplementary Data 1).

426 Microbiome community analysis. We analyzed the WMS data using mapping-based and

427 assembly-based hybrid methods PathoScope 2.0 (PS) ${ }^{65}$ and MG-RAST 4.0 (MR) ${ }^{8}$. In PS

428 analysis, a 'target' genome library was constructed containing all bacterial and archaeal

429 sequences from the NCBI Database

430 (https://en.wikipedia.org/wiki/National_Center_for_Biotechnology_Information) using the

431 PathoLib module. The reads were then aligned against the target libraries using the very sensitive

432 Bowtie 2 algorithm ${ }^{15-16}$ and filtered to remove the reads aligned with the cattle genome (bosTau8)

433 and human genome (hg38) as implemented in PathoMap (-very-sensitive-local -k 100 --score-

$434 \min \mathrm{L}, 20,1.0)$. Finally, the PathoID ${ }^{66}$ module was applied to obtain accurate read counts for

435 downstream analysis. In these samples, an average of 12.90 million aligned reads per sample

436 mapped to the target reference genome libraries $(96.24 \%)$ after filtering the cow and human

437 genome (Supplementary Data 1). The raw sequences were simultaneously uploaded in MR 
438 server (release 4.0) with proper embedded metadata and were subjected to the quality filter

439 containing dereplication and removal of host DNA by screening ${ }^{67}$ for taxonomical and functional

440 assignment.

441 Diversity analysis. Alpha diversity (diversity within samples) was estimated using the Shannon

442 index for both PS and MR reads output. To test beta diversity (differences in the organismal

443 structure) of the milk microbiome, a principal coordinate analysis (PCoA) was performed based

444 on weighted-UniFrac distances (for PS data) through Phyloseq $\mathrm{R}^{68}$, and Bray-Curtis dissimilarity

445 matrix (for MR data) ${ }^{69}$. In addition, non-metric multidimensional scaling (NMDS) on PS data

446 was also used for beta diversity analysis between the sample groups ${ }^{70}$. Taxonomic abundance

447 was determined by applying the "Best Hit Classification'" option using the NCBI database as a

448 reference with the following settings: maximum e-value of $1 \times 10^{-30}$; minimum identity of $95 \%$ for

449 bacteria, $60 \%$ for archaea and viruses, and a minimum alignment length of 20 as the set

450 parameters. The phylogenetic origin of the metagenomic sequences was projected against the

451 NCBI taxonomic tree and determined by the lowest common ancestor (LCA) with the same

452 cutoff mentioned above. Two phylogenetic trees consisting of 363 and 146 bacterial strains

453 respectively in $\mathrm{CM}$ and $\mathrm{H}$ metagenomes with $>80 \%$ taxonomic identity were constructed using

454 the neighbor-joining method in Clustal W (version 2.1) ${ }^{71}$, and FigTree (version 1.5.1) ${ }^{13}$.

455 Statistical analysis. The characteristics of cows with and without CM were compared using

456 Fisher's exact test for categorical variables, and Mann-Whitney U test for quantitative

457 variables ${ }^{21}$. The Shapiro-Wilk test was used to check normality of the data, and the non-

458 parametric test Kruskal-Wallis rank sum test was used to evaluate differences in the relative

459 percent abundance of taxa in $\mathrm{CM}$ and $\mathrm{H}$ groups. For the functional abundance profiling, the

460 statistical tests were applied at different KEGG and SEED subsystems levels in MR pipeline. 
461 Differences between the pipelines were evaluated using ANOVA and the Friedman rank sum 462 test. A significance level of alpha=0.05 was used for all tests ${ }^{8}$.

\section{Funding and Acknowledgements}

469 The Bangladesh Bureau of Educational Information and Statistics (BANBEIS), Ministry of

470 Education, Government of the People's Republic of Bangladesh (Grant No. LS2017313)

471 supported this work. The author M. Nazmul Hoque receives Fellowships from the Bangabandhu

472 Fellowship Trust, Ministry of Science and Technology, Government of the People's Republic of

473 Bangladesh. The authors also thank Keylie Gibson and Stephanie Warnken, PhD students at the

474 Computational Biology Institute, Milken Institute School of Public Health, the George

475 Washington University, USA for their for technical support in learning basic bioinformatics

476 operations.

\section{Data availability}

478 The raw sequence files have been submitted to NCBI database under BioProject PRJNA529353,

479 and can be accessed to the reviewers when they ask for it. All other relevant data supporting the 480 findings of the study are available in this article as Supplementary information files, or from the 481 corresponding author on request.

482 Author contributions 
483 M.N.H., M. S., A.M.A.M.Z.S. and M.A.H. conceived and designed the overall study, and

484 M.N.H. and R.A.C. carried out laboratory works including DNA extractions, quality control and

485 preparation for sequencing. M.A.H., R.A.C. and K.A.C. contributed reagents/materials/analysis

486 tools and sequencing. M.N.H. and A.I. conceived, designed and executed the bioinformatics

487 analysis and M.N.H. interpreted the results and prepared the manuscript. M.S., K.A.C., M.A.H

488 contributed intellectually to the interpretation and presentation of the results. Finally, all authors

489 have approved the manuscript for submission.

490

491 Competing interests: The authors of this study declare no competing interests.

492 References

493 1. Falentin, $H$. et al. Bovine teat microbiome analysis revealed reduced alpha diversity and 494 significant changes in taxonomic profiles in quarters with a history of mastitis. Front. $495 \quad$ Microbiol. 7, 480 (2016).

496 2. Aitken, S. L., Christine, M. C., \& Lorraine, M. S. Immunopathology of mastitis: insights into 497 disease recognition and resolution. J. Mammary Gland Biol. Neoplasia 16(4), 291-304 $498 \quad$ (2011).

499 3. Lin, S. et al. Mammary inflammatory gene expression was associated with reproductive stage 500 and regulated by docosahexenoic acid: in vitro and in vivo studies. Lipids Health Dis. 15(1), $501215(2016)$

502 4. Rinaldi, M., Li, R. W., \& Capuco, A. V. Mastitis associated transcriptomic disruptions in 503 cattle. Vet. Immun. Immunopath. 138(4), 267-279 (2010).

504 5. Patel, S. H. et al. Culture independent assessment of human milk microbial community in 505 lactational mastitis. Sci. Rep. 7(1), 7804 (2017). 
506 6. Kateete, D.P. et al. Prevalence and antimicrobial susceptibility patterns of bacteria from 507 milkmen and cows with clinical mastitis in and around Kampala, Uganda. PloS One 8(5), 508 p.e63413 (2013).

509 7. Catozzi, C. et al. The microbiota of water buffalo milk during mastitis. PloS One 12(9), $510 \quad \mathrm{e} 0184710(2017)$

511 8. D’Argenio, V., Giorgio, C., Vincenza, P., \& Francesco, S. Comparative metagenomic 512 analysis of human gut microbiome composition using two different bioinformatic pipelines. 513 BioMed Res. Int. 325340 (2014).

514 9. Cremonesi, P. et al. Milk microbiome diversity and bacterial group prevalence in a 515 comparison between healthy Holstein Friesian and Rendena cows. PLoS One 13(10), $516 \mathrm{e} 0205054$ (2018).

517 10. Salvetti, E. et al. Whole-metagenome-sequencing-based community profiles of Vitisvinifera 518 L. cv. Corvina berries withered in two post-harvest conditions. Front. Microbiol. 7, 937 519 (2016).

520 11. Bicalho M. L. S., Machado V. S., Higgins C. H., Lima F. S., \& Bicalho R. C. Genetic and 521 functional analysis of the bovine uterine microbiota. Part I: metritis versus healthy cows. $J$. 522 Dairy Sci.100 3850-3862 (2017).

523 12. Blackburn, M. R., \&Linda, F. T. Adenosine deaminase deficiency: unanticipated benefits 524 from the study of a rare immunodeficiency. The J. Immunol. 188(3), 933-935 (2012).

525 13. Stewart, R. D. et al. Assembly of 913 microbial genomes from metagenomic sequencing of 526 the cow rumen. Nat. Commun. 9(1), 870 (2018).

527 14. Vaibhav, D. B., Anju, P. K., Keyur, D. B., Navin, R. S., \& Chaitanya, G. J. Analysis of 528 virulence associated and antibiotic resistance genes of microbes in subclinical mastitis 529 affected cattle milk by pyrosequencing approach. J. Vet. Sci. Med. Diag. 2(2), 3 (2014). 
530 15. Oniciuc, E. et al. The Present and Future of Whole Genome Sequencing (WGS) and Whole

531 Metagenome Sequencing (WMS) for Surveillance of Antimicrobial Resistant

532 Microorganisms and Antimicrobial Resistance Genes across the Food Chain. Genes 9(5), 268

$533 \quad$ (2018).

534 16. Pärnänen, K. et al. Maternal gut and breast milk microbiota affect infant gut antibiotic 535 resistome and mobile genetic elements. Nat. Commun. 9(1), 3891 (2018).

536 17. Oikonomou, G. et al. Microbiota of cow's milk; distinguishing healthy, sub-clinically and $537 \quad$ clinically diseased quarters. PloS One 9(1), e85904 (2014).

538 18. Ranjan, R., Rani, A., Metwally, A., McGee, H. S., \& Perkins, D. L. Analysis of the 539 microbiome: Advantages of whole genome shotgun versus 16S amplicon sequencing. 540 Biochem. Biophys. Res. Commun. 469(4), 967-77 (2015).

541 19. Ganda, E. K. et al. Longitudinal metagenomic profling of bovine milk to assess the impact 542 of intramammary treatment using athird-generation cephalosporin. Sci. Rep. 6, 37565, 543 doi:10.1038/srep37565 12 (2016).

544 20. Bhatt, V. D. et al. Milk microbiome signatures of subclinical mastitis-affected cattle analysed 545 by shotgun sequencing. J. Appl. Microbiol. 112(4), 639-650 (2012).

546 21. Wang, J. et al. Metagenomic sequencing reveals microbiota and its functional potential $547 \quad$ associated with periodontal disease. Sci. Rep. 3, 1843 (2013).

548 22. Zong, Z. The complex genetic context of blaPER-1 flanked by miniature inverted-repeat 549 transposable elements in Acinetobacter johnsonii. PloS One 9(2), e90046 (2014).

550 23. Montaña, S. et al. The genetic analysis of an Acinetobacter johnsonii clinical strain 551 evidenced the presence of horizontal genetic transfer. PloS One 11(8), e0161528 (2016).

552 24. Carro, L. et al. Genome-based classification of micromonosporae with a focus on their 553 biotechnological and ecological potential. Sci. Rep. 8(1), 525 (2018).

554 25. Nusrat, J. MALDI-TOF: A Rapid Identification of Dairy Pathogens. (2017). 
26. Meng, L., Zhang, Y., Liu, H., Zhao, S., Wang, J., \& Zheng, N. Characterization of

556 Pseudomonas spp. and Associated Proteolytic Properties in Raw Milk Stored at Low

557 Temperatures. Front. Microbiol. 8, 2158, (2017).

558 27. Sela, S., Hammer-Muntz, O., Krifucks, O., Pinto, R., Weisblit, L., \& Leitner, G. Phenotypic 559 and genotypic characterization of Pseudomonas aeruginosa strains isolated from mastitis 560 outbreaks in dairy herds. J. Dairy Res. 74, 425-429 (2007).

561 28. Podder, M.P., Rogers, L., Daley, P.K., Keefe, G.P., Whitney, H.G., \& Tahlan, K. Klebsiella 562 species associated with bovine mastitis in Newfoundland. PloS One 9(9), e106518 (2014).

563 29. Maga, E. A., Weimer, B. C., \& Murray, J. D. Dissecting the role of milk components on gut 564 microbiota composition. Gut Microbes 4(2), 136-9 (2013).

565 30. Ma, C. et al. Cow-to-mouse fecal transplantations suggest intestinal microbiome as one cause 566 of mastitis. Microbiome 6(1), 200 (2018).

567 31. Modi, S. et al. Prevalence of Campylobacter species in milk and milk products, their $568 \quad$ virulence gene profile and anti-bio gram. Vet. World 8(1), 1 (2015).

569 32. Bianchini, V. et al. Prevalence in bulk tank milk and epidemiology of Campylobacter jejuni $570 \quad$ in dairy herds in Northern Italy. Appl. Environ. Microbiol. 03784 (2014).

571 33. Gomes, F., Maria, J. S., \& Mariana, H. Bovine mastitis disease/pathogenicity: evidence of 572 the potential role of microbial biofilms. Pathogens Dis. 74(3), (2016).

573 34. Hoque, M. N. et al. Molecular characterization of Staphylococcus aureus strains in bovine 574 mastitis milk in Bangladesh. Int. J. Vet. Sci. Med. 6, 53-60 (2018).

575 35. Leimbach, A. et al. Whole-genome draft sequences of six commensal fecal and six mastitis576 associated Escherichia coli strains of bovine origin. Genome Announc. 4(4), e00753-16 $577 \quad$ (2016).

578 36. Lurie-Weinberger, \& Gophna, M. N. Archaea in and on the human body: health implications $579 \quad$ and future directions. PLoS Pathog. 11(6), e1004833 (2015). 
37. Marcó, M. B., Moineau, S., \& Quiberoni, A. Bacteriophages and dairy fermentations.

$581 \quad$ Bacteriophage 2, 149-158 (2012).

582 38. Lagier, J. C. et al. Many more microbes in humans: enlarging the microbiome repertoire.

583 Clinic. Infect. Dis. 65(1), S20-S29 (2017).

584 39. Shkoporov, A. N., \& Hill, C. Bacteriophages of the Human Gut: The "Known Unknown" of 585 the Microbiome. Cell Host Microbe 25(2), 195-209 (2019).

586 40. Riaz, A., Kifayatullah, M. H., \& Naeem, A. Recent Understanding of the Classification and 587 Life Cycle of Herpesviruses: A Review. Sci. Letters 5(2), 195-207 (2017).

588 41. Alpay, G., \& Ye $\square$ İlbağ, K. The roles of viruses in mastitis. Uludag Univ. Vet. 28(1), 39-46 589 (2009).

590 42. Li, N. et al. Variation in raw milk microbiota throughout 12 months and the impact of $591 \quad$ weather conditions. Sci. Rep. 8(1), 2371 (2018).

592 43. Frese, S.A., Parker, K., Calvert, C.C., \& Mills, D.A. Diet shapes the gut microbiome of pigs 593 during nursing and weaning. Microbiome 3, 2 (2015).

594 44. Green, J., Matthew, D. R., \& Laura, J. S. Transcriptional regulation of bacterial virulence 595 gene expression by molecular oxygen and nitric oxide. Virulence 5(8), 794-809 (2014).

596 45. Li, X., Ding, X. Z., Wan, Y. L., Liu, Y. M., \& Du, G.Z. Comparative proteomic changes of 597 differentially expressed whey proteins in clinical mastitis and healthy yak cows. Genet. Mol. 598 Res. 13(3), 6593-6601 (2014).

599 46. Rajkumari, J., L. P. S., \& Piyush, P. Genomic insights of aromatic hydrocarbon degrading 600 Klebsiella pneumoniae AWD5 with plant growth promoting attributes: a paradigm of soil 601 isolate with elements of biodegradation. 3 Biotech 8(2), 118 (2018).

602 47. Eloe-Fadrosh, E. A., \& Rasko, D. A. The human microbiome: from symbiosis to 603 pathogenesis. Annu. Rev. Med. 64, 145-163 (2013). 
604 48. Matilla, M.A., \& Krell, T. The effect of bacterial chemotaxis on host infection and 605 pathogenicity. FEMS Microbiol. Reviews 42(1), 24 (2017).

606 49. Koul, H.K., Pal, M., Koul, S. Role of p38 MAP kinase signal transduction in solid tumors.

607 Genes Cancer 4(9-10), 342-59 (2013).

608 50. Song, H., Ki, S. H., Kim, S. G., Moon, A. Activating transcription factor 2 mediates matrix 609 metalloproteinase-2 transcriptional activation induced by p38 in breast epithelial cells. $610 \quad$ Cancer Res. 66(21), 10487-96 (2006).

611 51. Long, E. et al. Escherichia coli induces apoptosis and proliferation of mammary cells. Cell 612 Death Differ.8, 808-816 9 (2001).

613 52. Sakamoto, T., Daigo, N., \& Motoharu, S. Targeting the warburg effect, which arises in tumor 614 cells expressing membrane-type-1 matrix metalloproteinase. J. Biological Chem. jbc-M110 615 (2011).

616 53. Li, C. et al. UFL1 Alleviates Lipopolysaccharide-Induced Cell Damage and Inflammation via 617 Regulation of the TLR4/NF-кB Pathway in Bovine Mammary Epithelial Cells. Oxid. Med. 618 Cell. Longev. ID 6505373, 1-17 (2019).

619 54. Gomes, F, Saavedra, M. J. \& Henriques, M. Bovine mastitis disease/pathogenicity: evidence 620 of the potential role of microbial biofilms. Patho. Dis. 74(3), 1-7 (2016).

621 55. Hibbing, M. E., Fuqua, C., Parsek, M. R., \& Peterson, S. B. Bacterial competition: surviving 622 and thriving in the microbial jungle. Nat. Rev. Microbiol. 8, 15-25 (2010).

623 56. Farzaneh, M., Haghkhah, M., Nazifi, S., Lari, M.A., \& Fani, M.M. Evaluation of milk 624 adenosine deaminase activities in dairy cattle with subclinical mastitis and their correlation 625 with milk quality. Iranian J. Vet. Clin. Sci. 12(1), (2018).

626 57. Baba, K. et al. Adenosine deaminase activity is a sensitive marker for the diagnosis of 627 tuberculous pleuritis in patients with very low CD4 counts. PloS One 3(7), e2788 (2008). 
628 58. Moreno, E. et al. Molecular evidence of adenosine deaminase linking adenosine A2A

629 receptor and CD26 proteins. Frontiers Pharmaco. 9, 106 (2018).

630 59. Sampson, T. R., \& Weiss, D. S. Alternative Roles for CRISPR/Cas Systems in Bacterial

631 Pathogenesis. PLoS Pathog. 9(10), e1003621 (2013).

632 60. Cheng, D. et al. Prevalence and Isoforms of the Pathogenicity Island ETT2 Among

633 Escherichia coli Isolates from Colibacillosis in Pigs and Mastitis in Cows. Current

$634 \quad$ Microbiol. 64(1), 43-49 (2011).

635 61. Abeles, S. R., \& Pride, D. T. Molecular bases and role of viruses in the human microbiome.

$636 \quad$ J. Molecular Biol. 426(23), 3892-3906 (2014).

637 62. Cheng, J. et al. Antimicrobial resistance profiles of 5 common bovine mastitis pathogens in

638 large Chinese dairy herds. J. Dairy Sci. 102, 1-11 (2018).

639 63. Hoque, M. N. et al. Different screening tests and milk somatic cell count for the prevalence 640 of subclinical bovine mastitis in Bangladesh. Trop. Anim. Health Prod. 47(1), 79-86 (2015).

641 64. Head, S. R. et al. Library construction for next-generation sequencing: overviews and 642 challenges. Biotechniques 56(2), 61-77 (2014).

643 65. Hong, C. et al. PathoScope 2.0: a complete computational framework for strain identification 644 in environmental or clinical sequencing samples. Microbiome 2(1), 33(2014).

645 66. Francis, O. E. et al. Pathoscope: species identification and strain attribution with 646 unassembled sequencing data. Genome Res. 23(10), 1721-1729 (2013).

647 67. Zheng, W. et al. Metagenomic sequencing reveals altered metabolic pathways in the oral 648 microbiota of sailors during a long sea voyage. Sci. Rep. 5, 9131 (2015).

649 68. McMurdie, P. J. \& Susan, H. Phyloseq: an R package for reproducible interactive analysis 650 and graphics of microbiome census data. PloS One 8(4), e61217 (2013). 
651 69. Janssens, P. L. H. R. et al. Long-term green tea supplementation does not change the human

652 gut microbiota. PloS One 11(4), e0153134 (2016).

653 70. Guan, Y. et al. Comparison of the gut microbiota composition between wild and captive sika

654 deer (Cervus Nippon hortulorum) from feces by high-throughput sequencing. AMB Express

$655 \quad \mathbf{7 ( 1 )}, 212(2017)$.

656 71. Lugli, G.A. et al. Comparative genomic and phylogenomic analyses of the

657 Bifidobacteriaceae family. BMC Genomics 18(1), 568 (2017).

658

659

660 
A

PathoScope Species Level

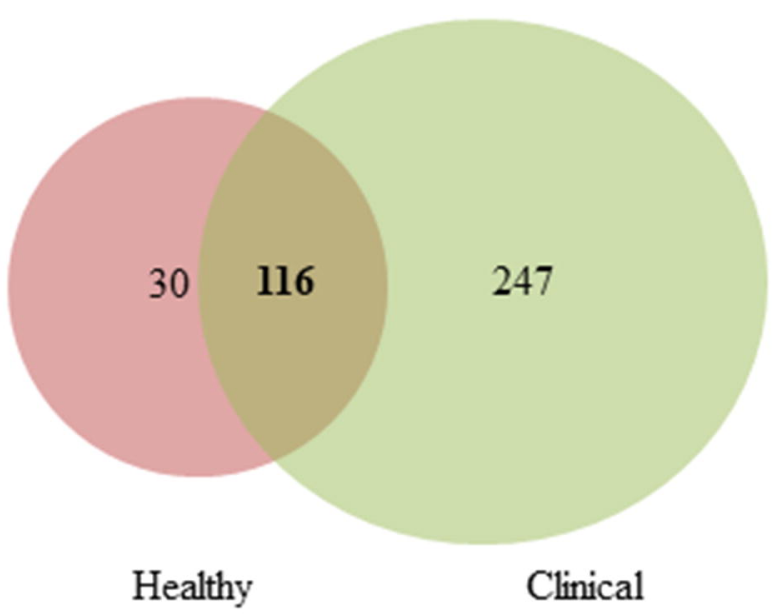

D

Taxa Distribution at Genus Level Archaea

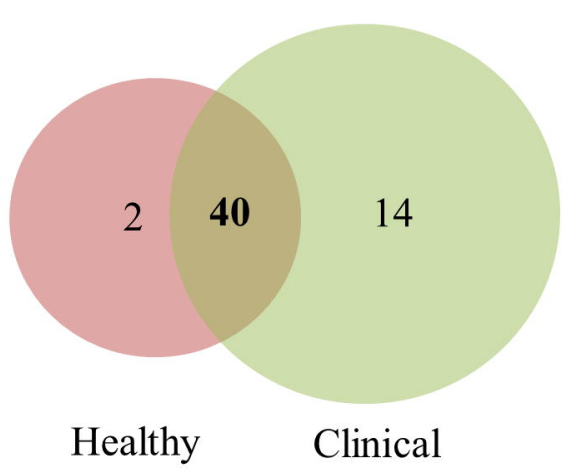

B

MG-RAST Genus Level

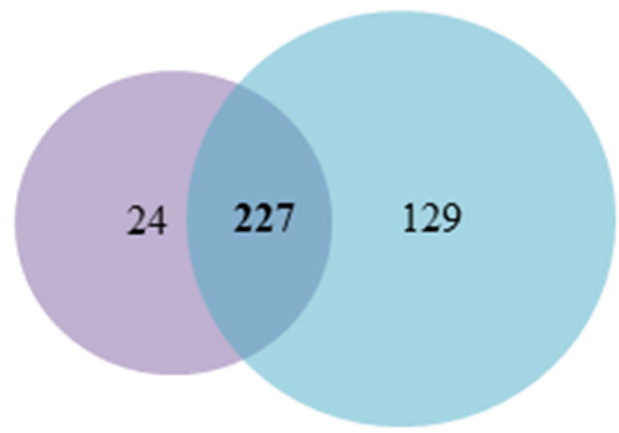

Healthy

Clinical
C

Taxa Distribution at Genus Level

PathoScope $\cap$ MG-RAST

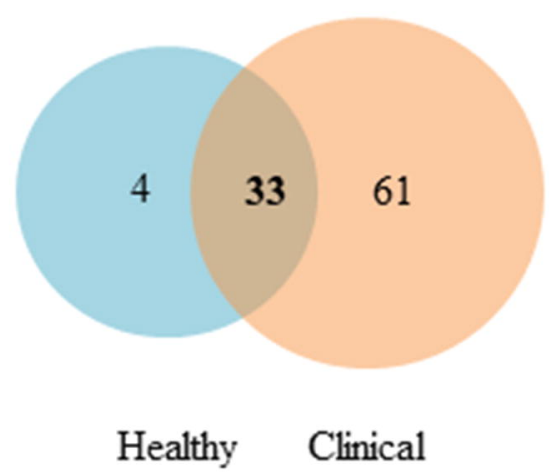

$\mathbf{E}$

Taxa Distribution at Genus Level Virus

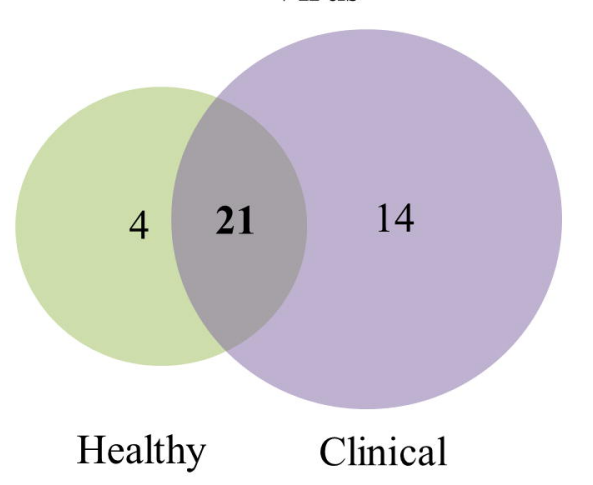




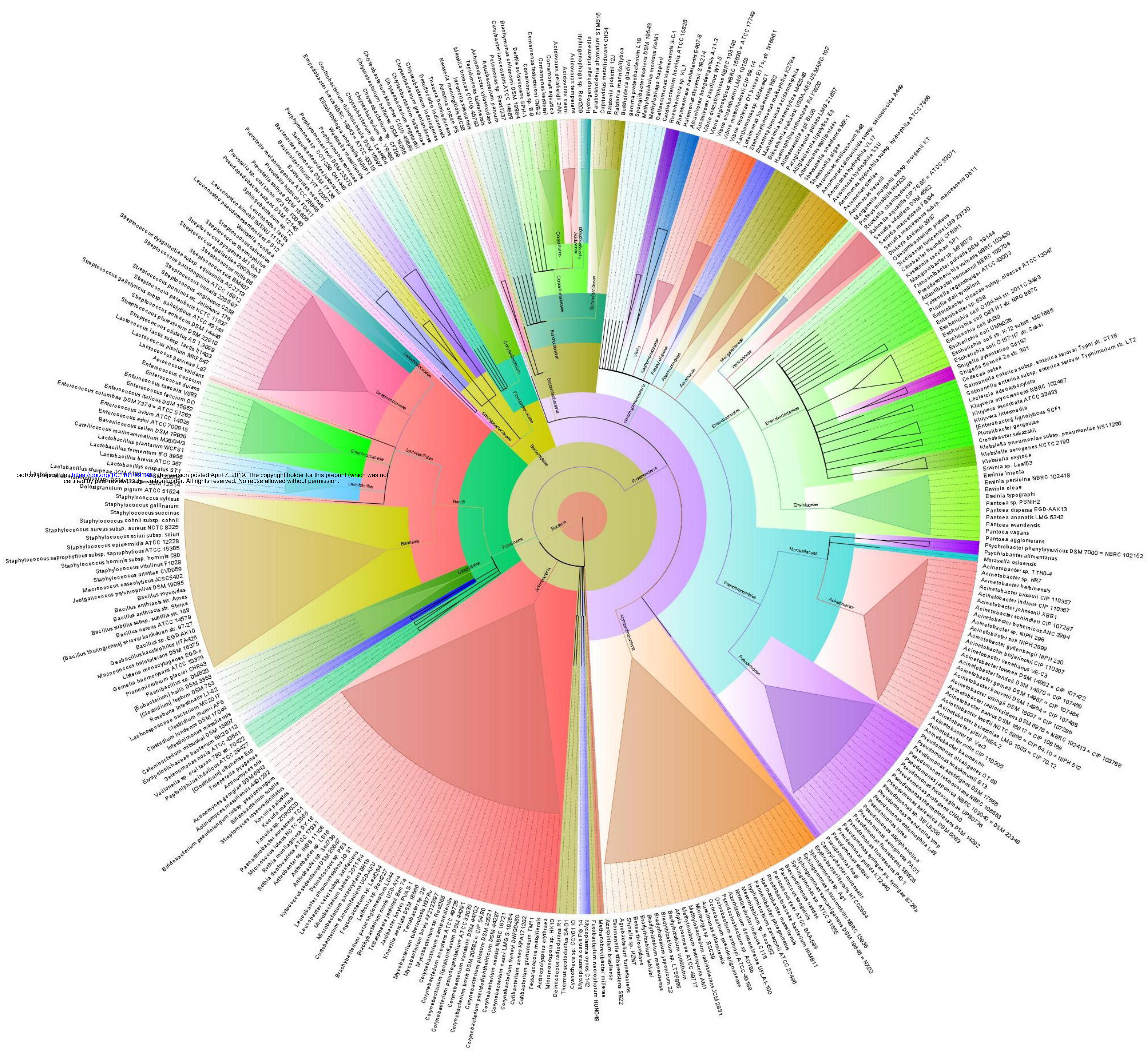




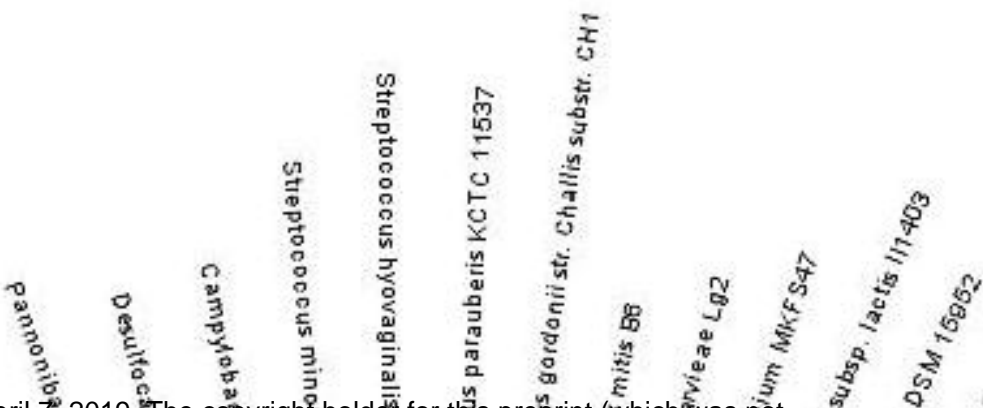

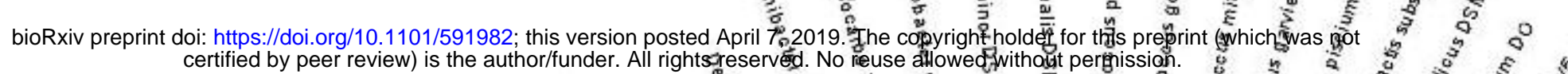

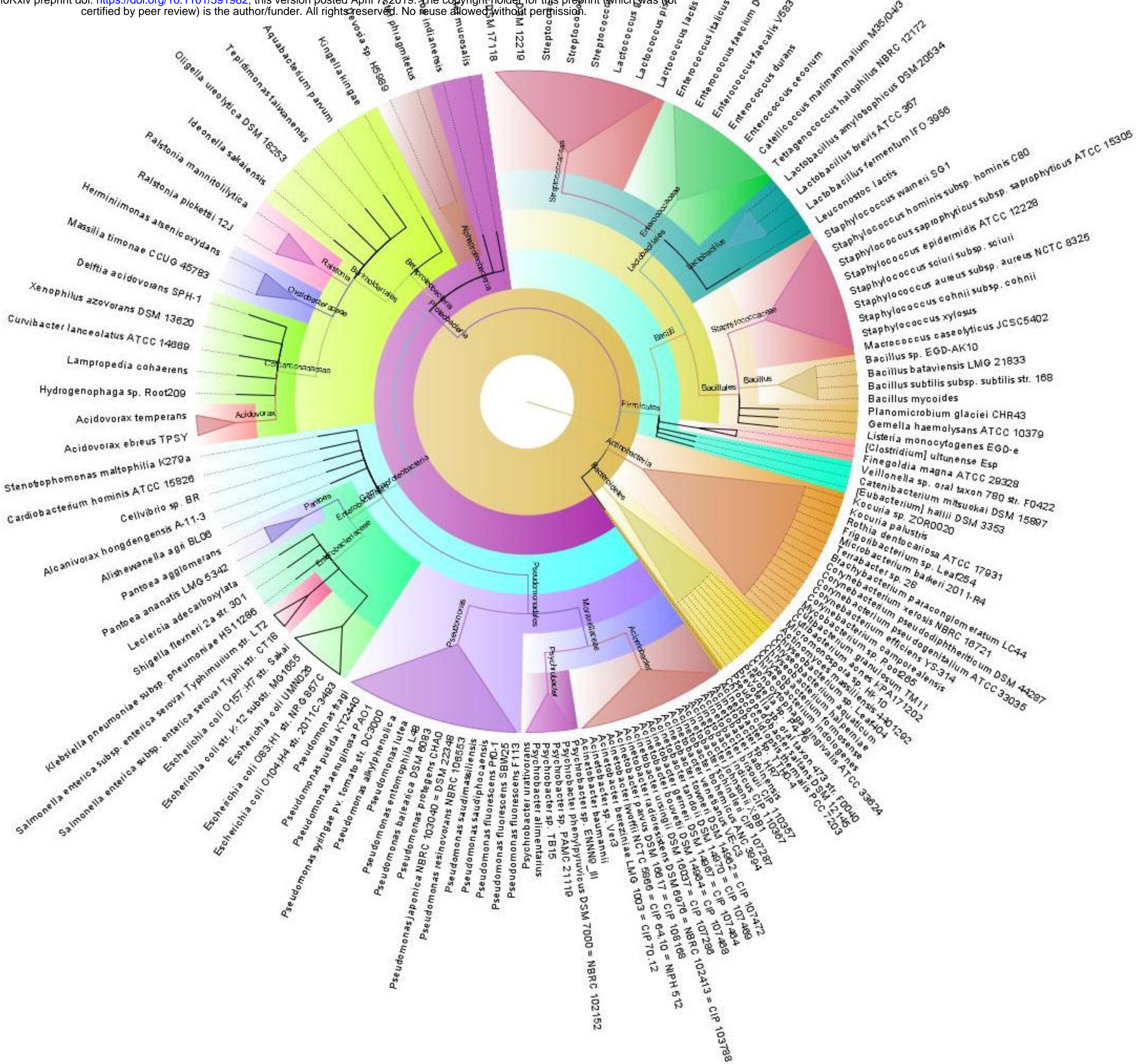



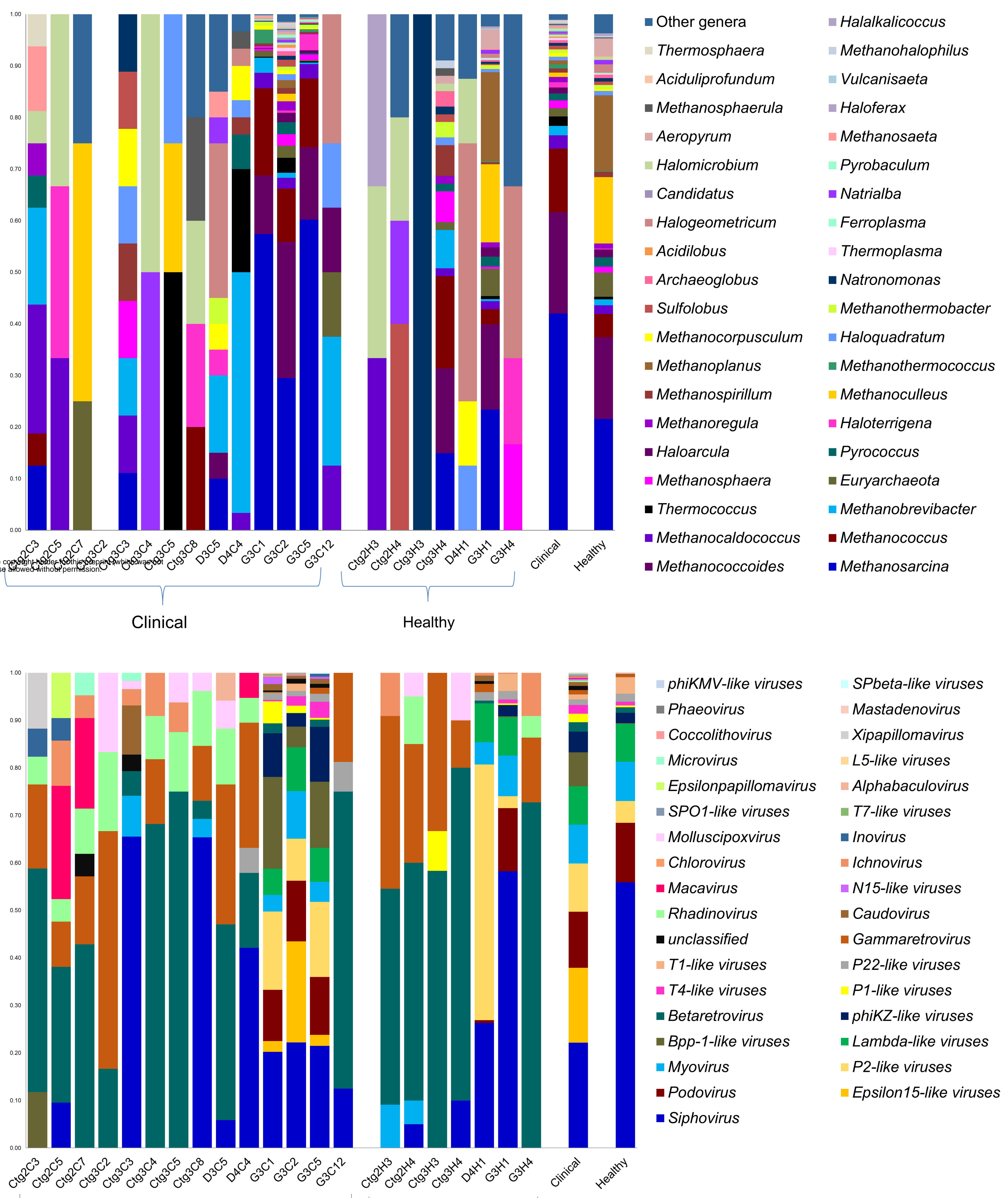

Clinical

Healthy 


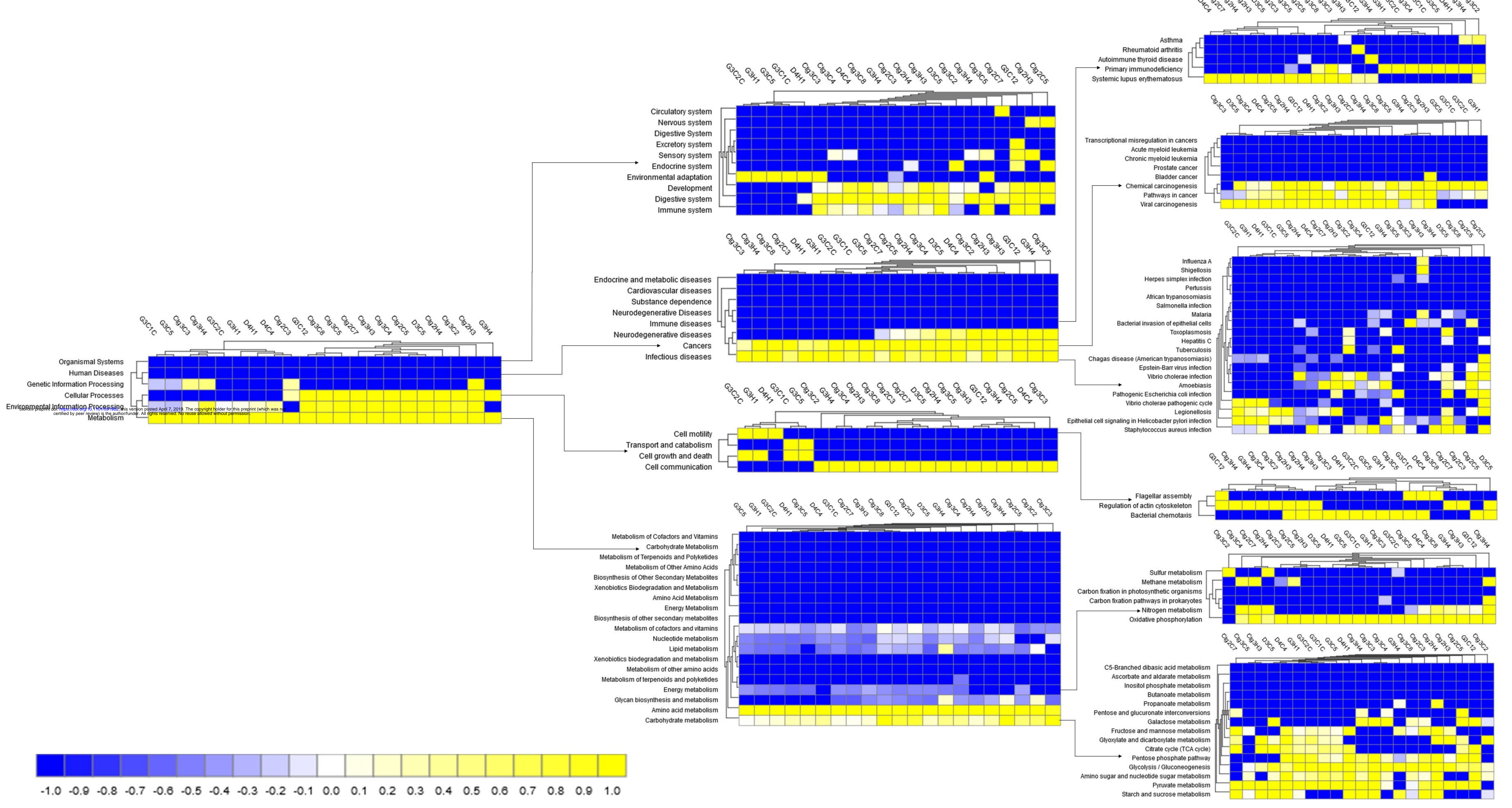




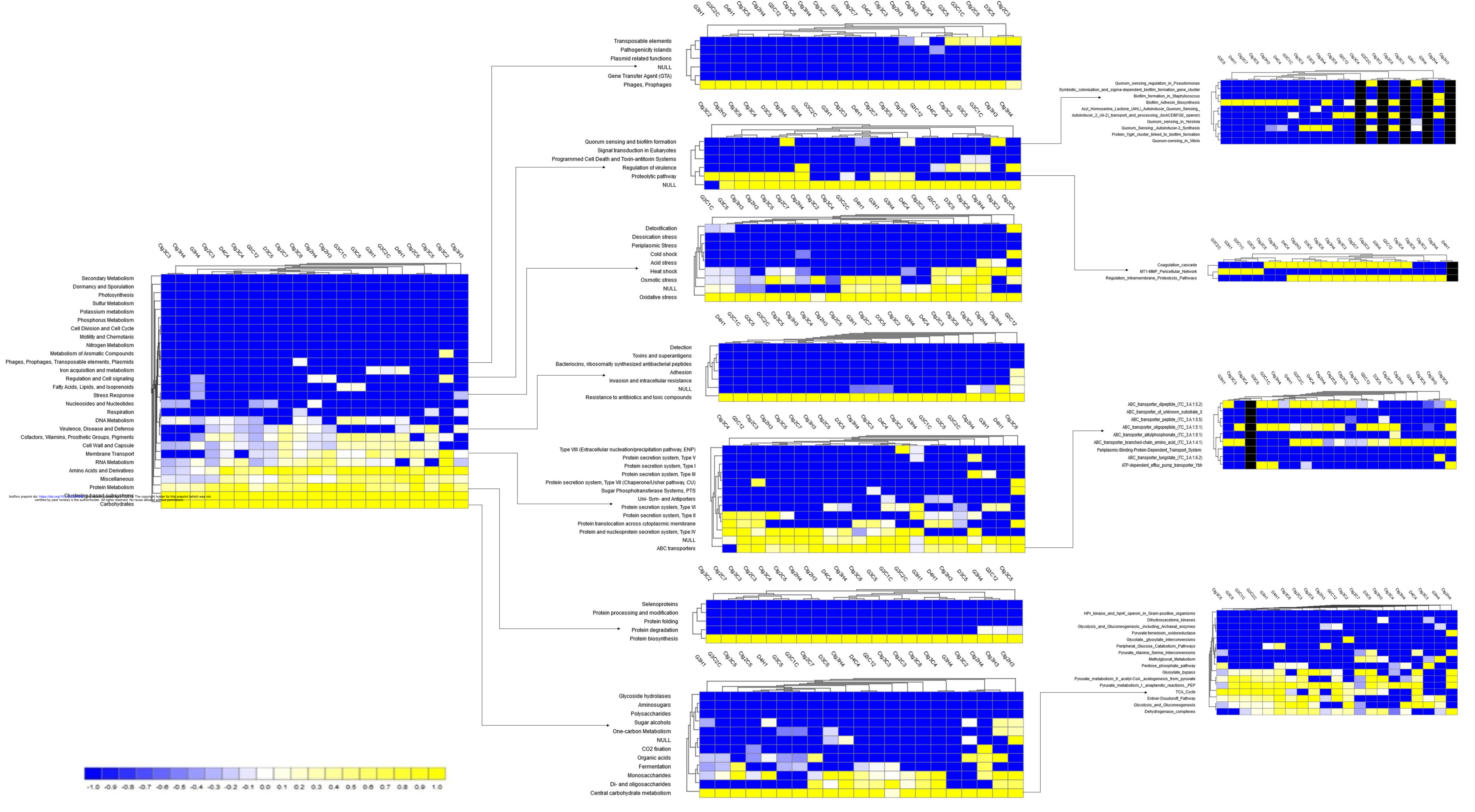


Multidrug Resistance Efflux Pumps

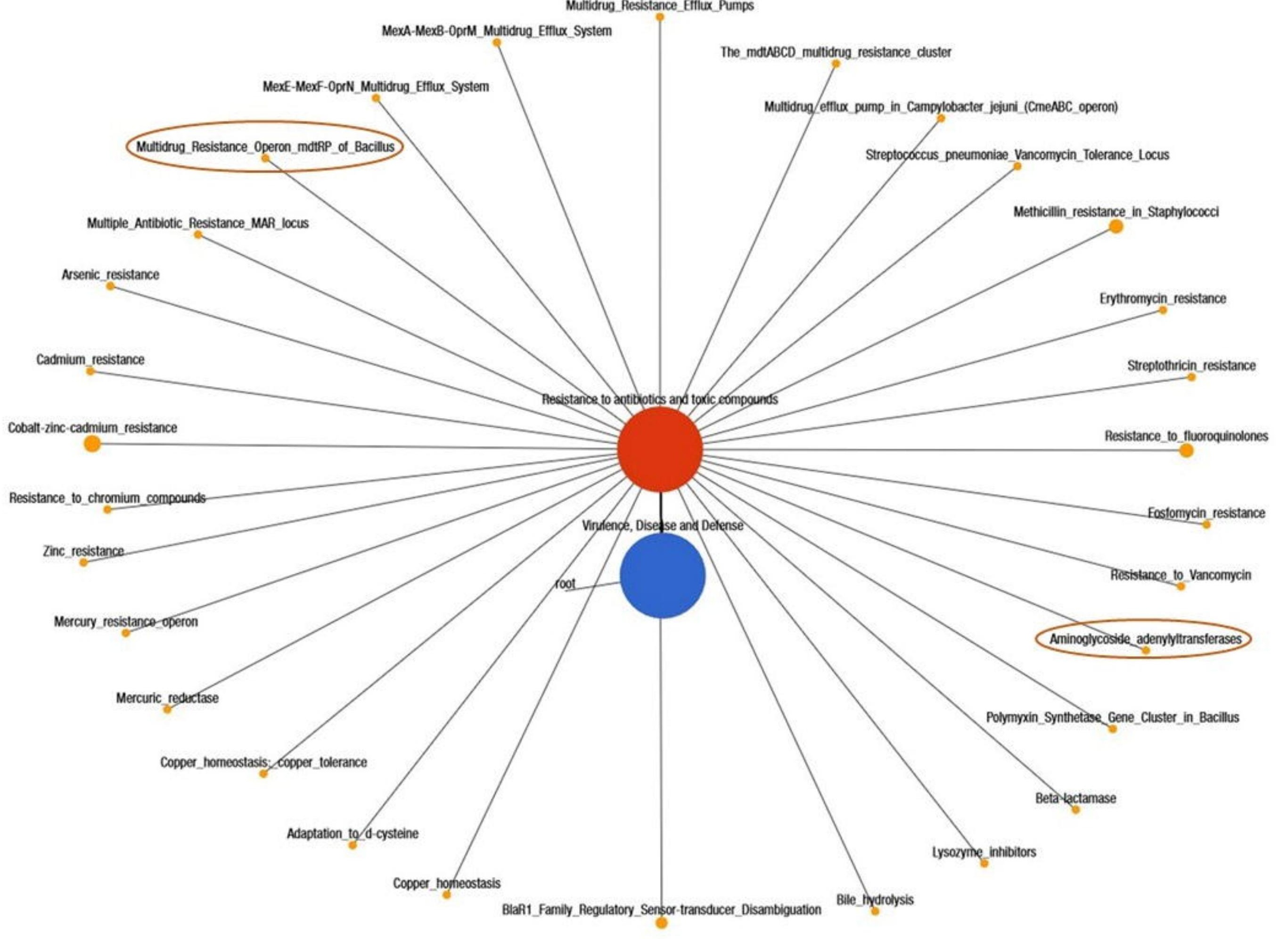



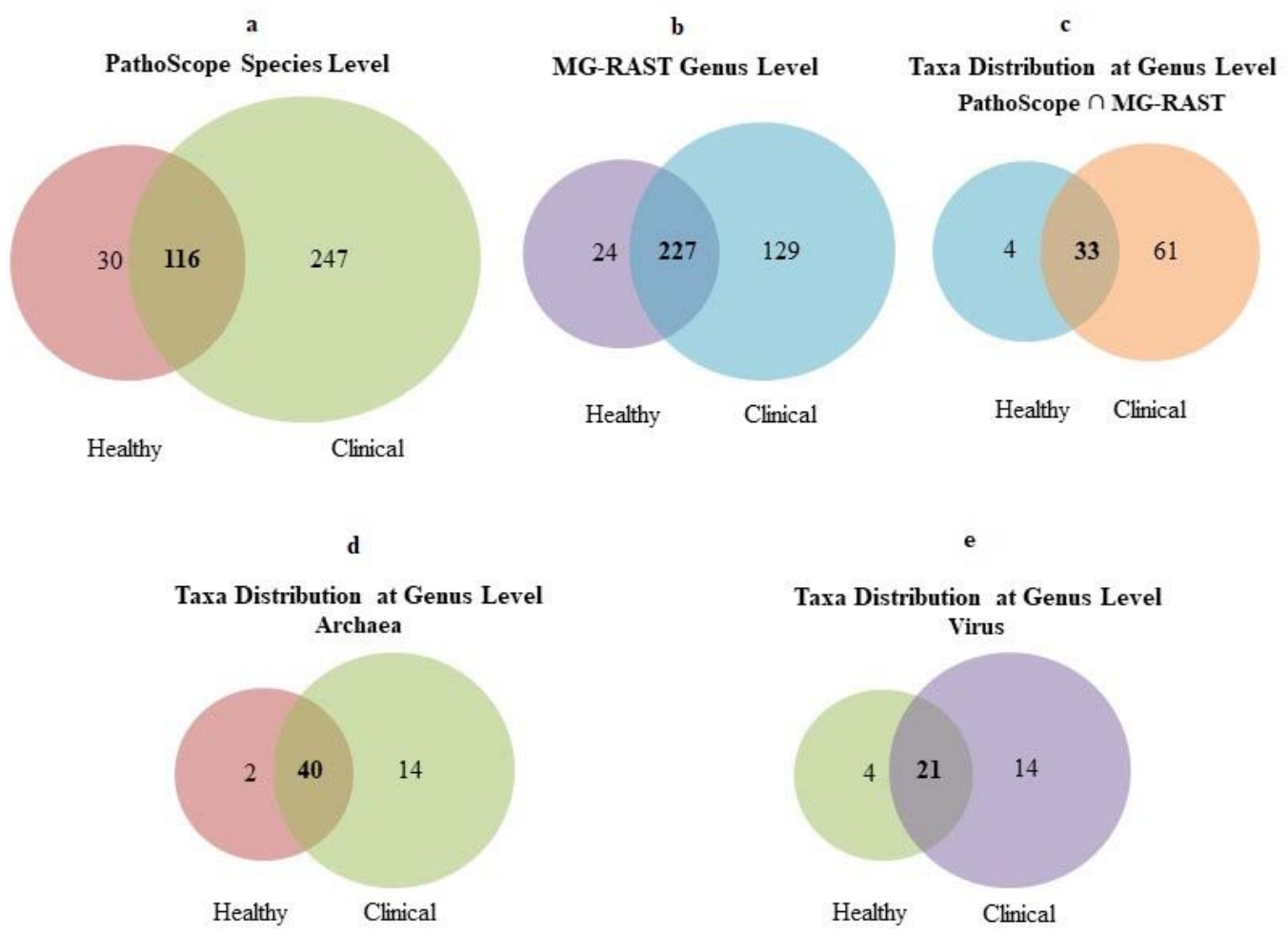

Fig. 1 Venn diagrams representing the core unique and shared microbiomes of bovine clinical mastitis (CM) and healthy $(\mathrm{H})$ milk. a Top Left: Venn diagram comparison of bacteria at strain level by PathoScope (PS), b Top Middle: Venn diagram showing unique and shared bacterial genera by MG-RAST (MR), c Top Right: Shared and unique bacterial genera distribution between PS and MR, d Bottom Left: \& e Bottom Right: Venn diagrams representing unique and shared Archaeal and viral genera respectively found in bovine milk as analysed with MR pipeline. Microbiotas sharing between the conditions are indicated by bold colored. 


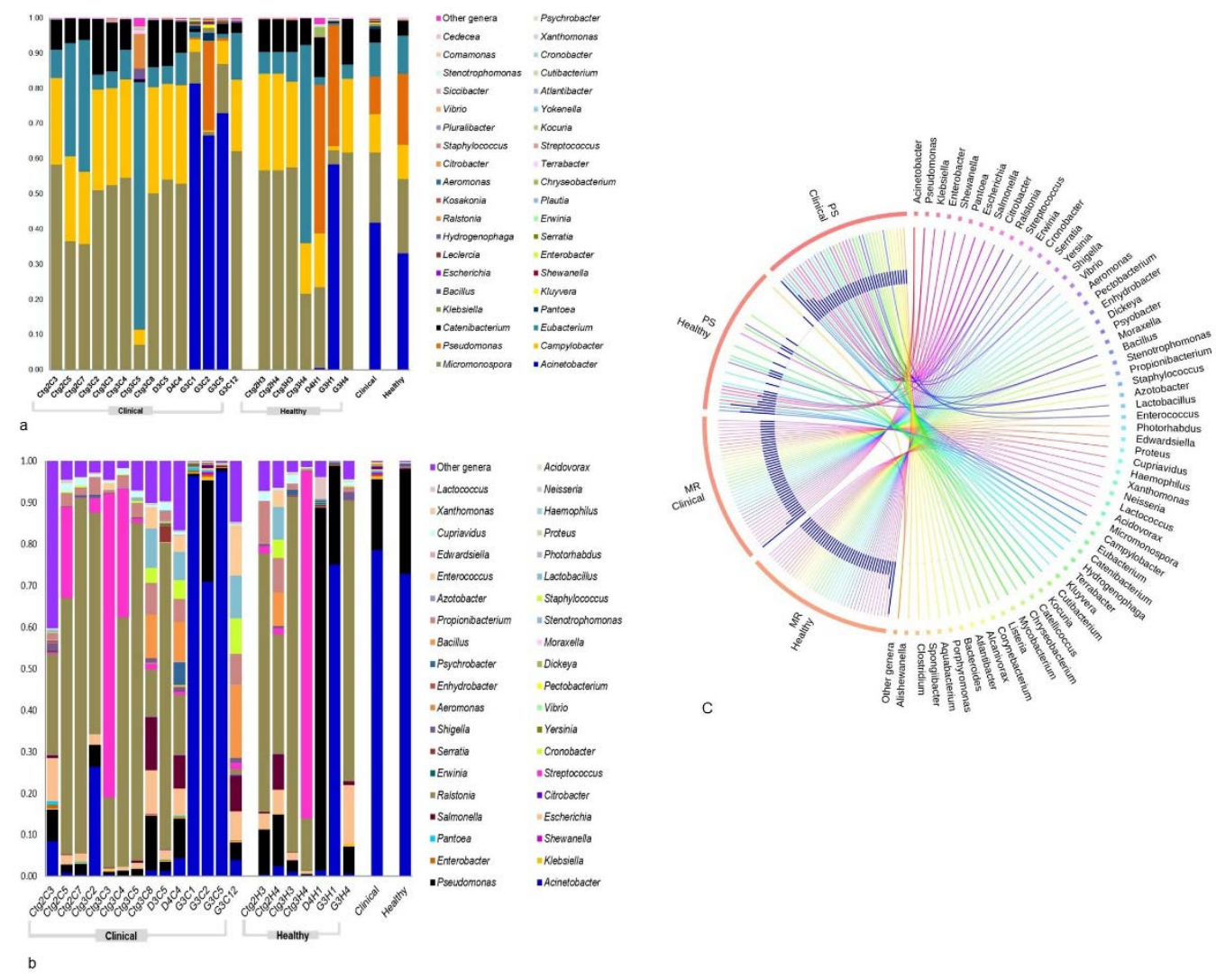

Fig. 2 Taxonomic profile of 40 most abundant bacterial genera in bovine clinical mastitis (CM) and healthy $(\mathrm{H})$ milk samples. a Top Left: abundance through PathoScope (PS) and b Bottom Left: through MG-RAST (MR) analyses. The 39 most abundant genera are sorted by descending order of the relative abundance in 21 samples, with the remaining genera grouped into the 'Other genera'. Each stacked bar plot represents the abundance of bacteria in each sample of the corresponding category, where the last two bar plots depict overall relative abundance of bacterial genera between CM and H samples, respectively. c Middle Right: The circular plot illustrates the relative abundance of top 40 bacterial genera in CM and H milk samples analysed through PS and MR. Taxa in both metagenomes are represented by different colored ribbons both tools. The relative abundancies are illustrated by the sizes of each color segment in the outer circle and the inner blue colored bars. Part of the microbiome is shared by both sample categories (CM-H milk), and part is analytic tool specific (PS-MR). Notable differences between the bacterial populations are those where the taxon is abundant in CM samples and effectively undetected in the H milk. Sample names: suffix ends with $\mathrm{C}$ refers to clinical (CM) and that ends with $\mathrm{H}$ refers to healthy $(\mathrm{H})$ milk samples. 


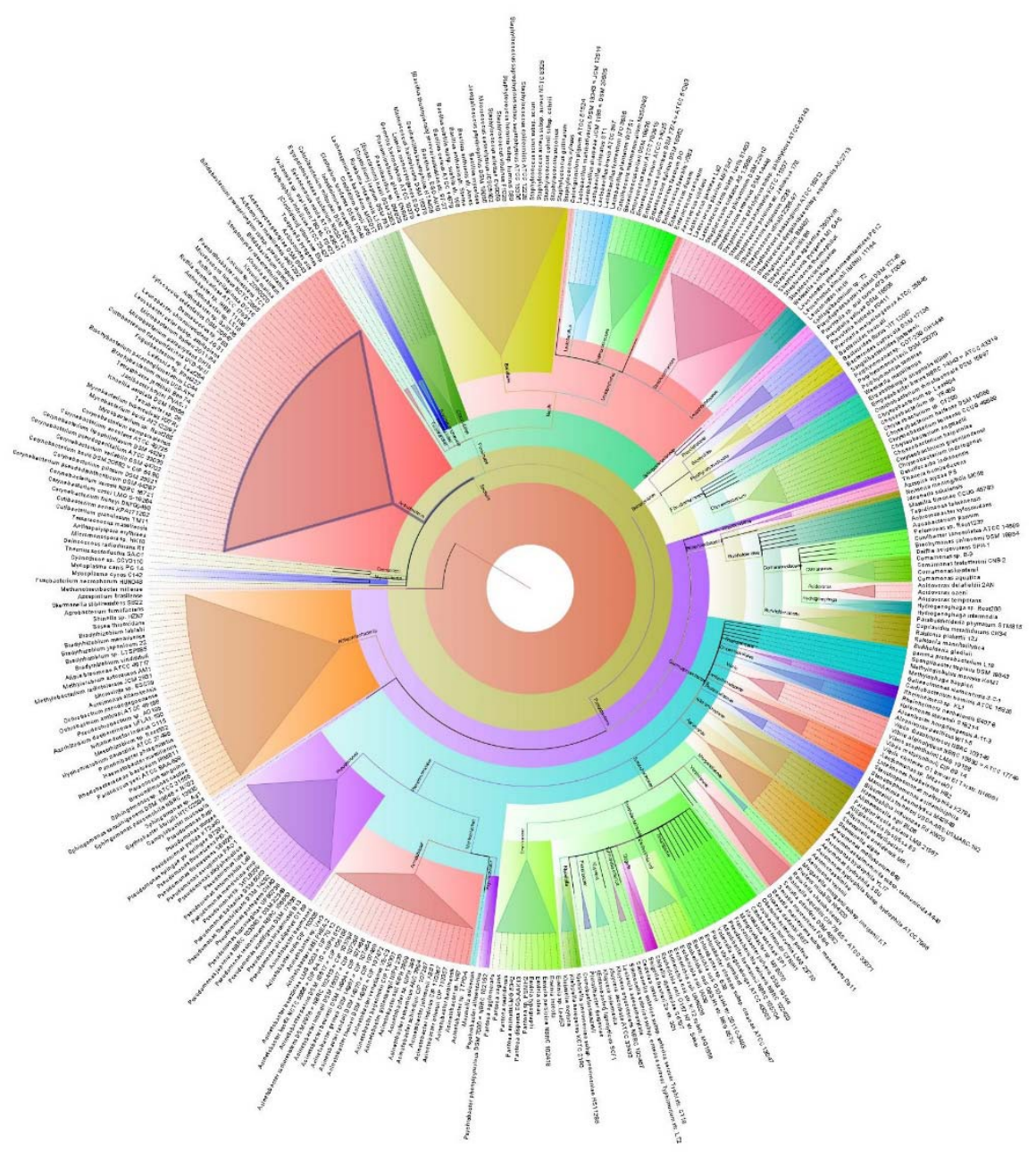

Fig. 3 Taxonomic representation of unique microbiota associated to the bovine clinical mastitis (CM) milk at strain level. Sequences are assigned to different taxonomic index in PathoScope analysis using minimum identity of $95 \%$ and minimum alignment length 20 as cutoff parameters, and the circular phylogenetic tree is constructed based on the neighbor-joining algorithm using FigTree. The round tree illustrates 363 unique strains of bacteria in CM milk metagenomes. The inner circle represents the root of the microbiome defined as bacteria present in all samples. The outer circles represent different strains of bacteria is defined as species (with different strains) present in $>50 \%$ of samples of the corresponding groups. For the outer circles, the width of a segment is proportional to the observed incidence for that species. Different colors are assigned according to the taxonomic ranks of the bacteria. The species and/or strains in the phylogenetic tree are also available in supplementary Data 2. 


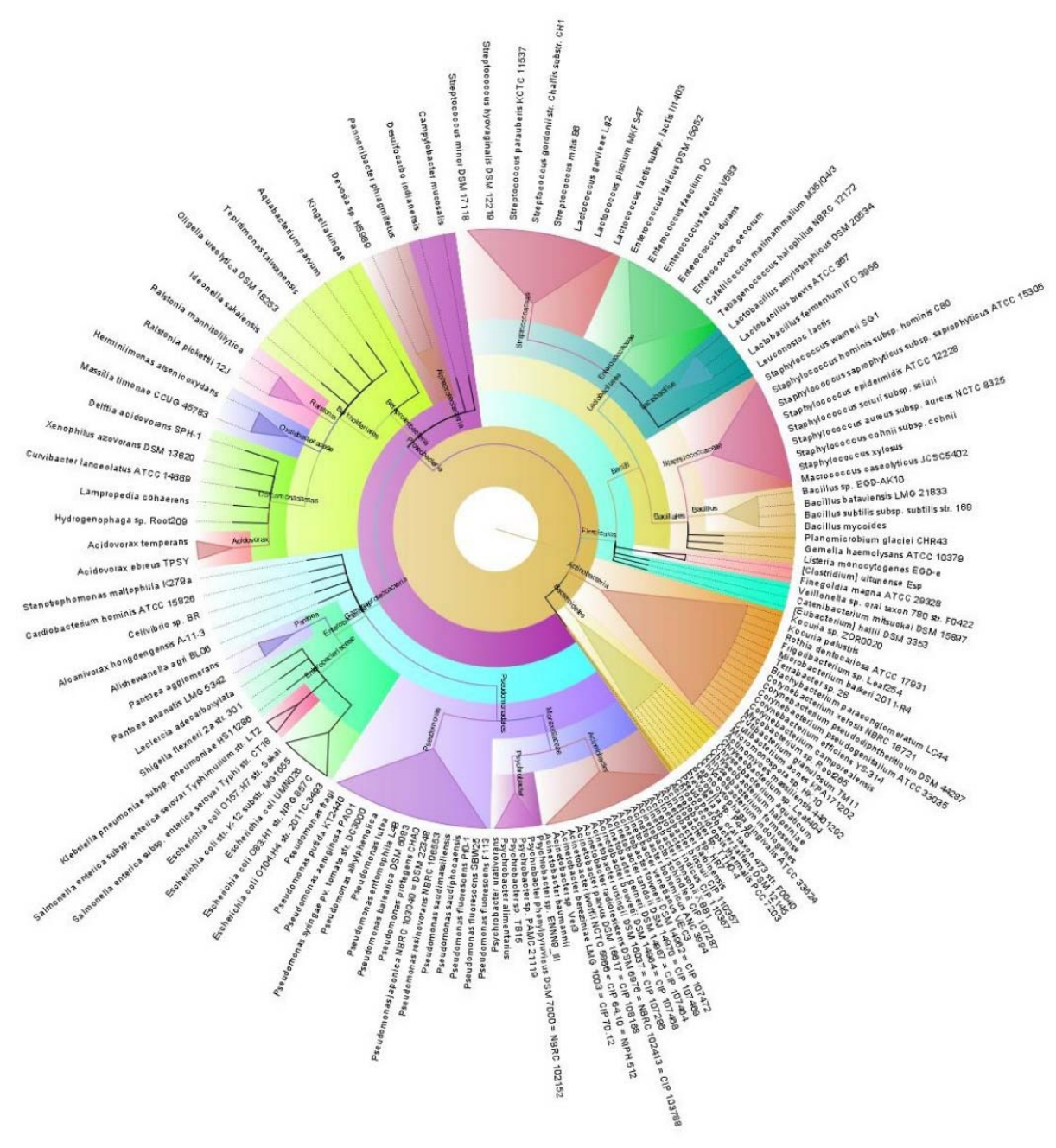

Fig. 4 Taxonomic representation of unique microbiota associated to the bovine clinical mastitis $(\mathrm{CM})$ and health $(\mathrm{H})$ milk at strain level. Sequences are assigned to different taxonomic index in PathoScope analysis using minimum identity of $95 \%$ and minimum alignment length 20 as cutoff parameters, and the circular phylogenetic tree is constructed based on the neighbor-joining algorithm using FigTree. The round tree illustrates 146 unique strains of bacteria in $\mathrm{H}$ milk metagenomes. The inner circle represents the root of the microbiome defined as bacteria present in all samples. The outer circles represent different strains of bacteria is defined as species (with different strains) present in $>50 \%$ of samples of the corresponding groups. For the outer circles, the width of a segment is proportional to the observed incidence for that species. Different colors are assigned according to the taxonomic ranks of the bacteria. The species and/or strains in the phylogenetic tree are also available in supplementary Data 2. 


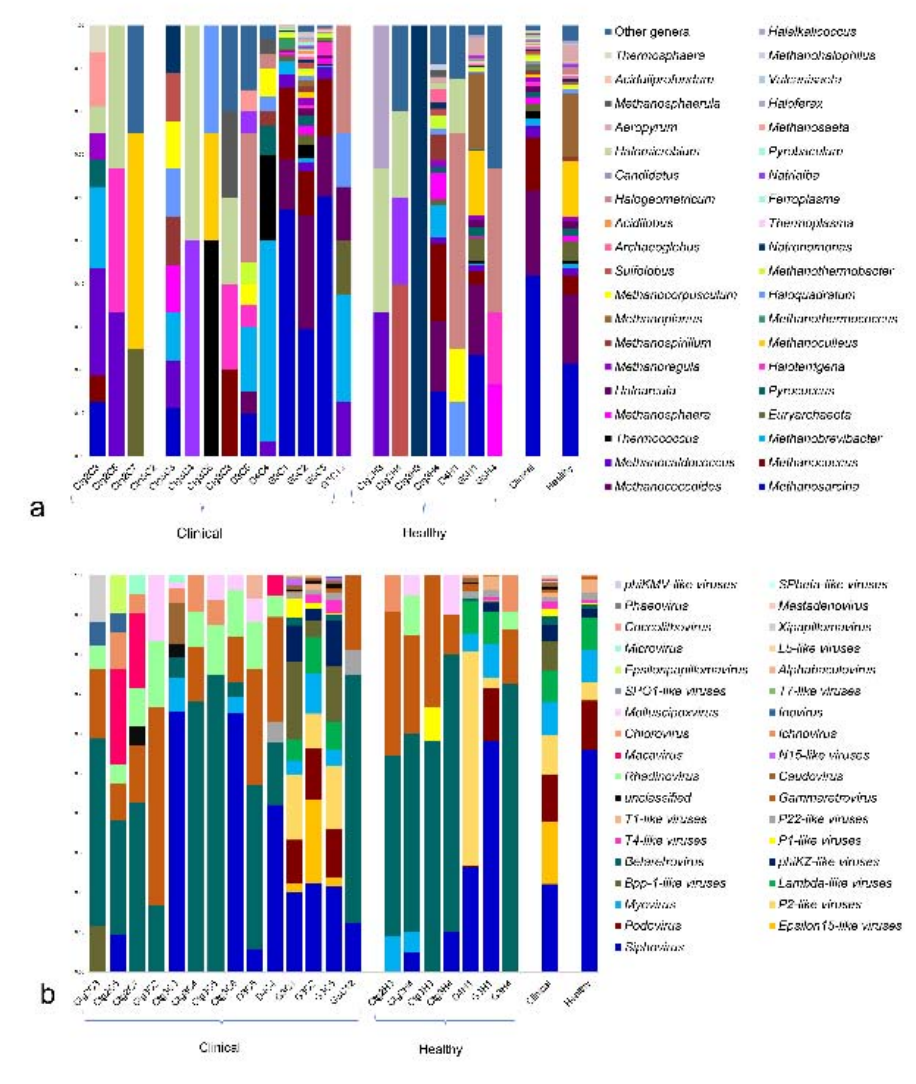

Fig. 5 Taxonomic abundance of top 40 archaeal and viral genera from the reads count of MR output. a Top: Archaeal genera are found in 20 samples, and absent in one clinical sample (Ctg3C2). The 39 most abundant archaeal genera are sorted by descending order of the relative abundance, with the remaining genera keeping into the 'Other genera'. b Bottom: Taxonomic distribution of 35 viral genera detected in all of the 21 samples of clinical $(\mathrm{CM})$ and healthy $(\mathrm{H})$ milk metagenomes. The most abundant viral genera are sorted by descending order of the relative abundance. Each stacked bar plot represents the abundance of archaea and viruses in each sample of the corresponding category, where the last two bar plots depict overall relative abundance of archaeal and viral genera in both metagenome groups. Notable differences between the archaeal and viral populations are those where the taxon is abundant in clinical samples and effectively undetected in the healthy milk. Sample names: suffix ends with $\mathrm{C}$ refers to clinical $(\mathrm{CM})$ and that ends with $\mathrm{H}$ refers to healthy $(\mathrm{H})$ milk samples. 


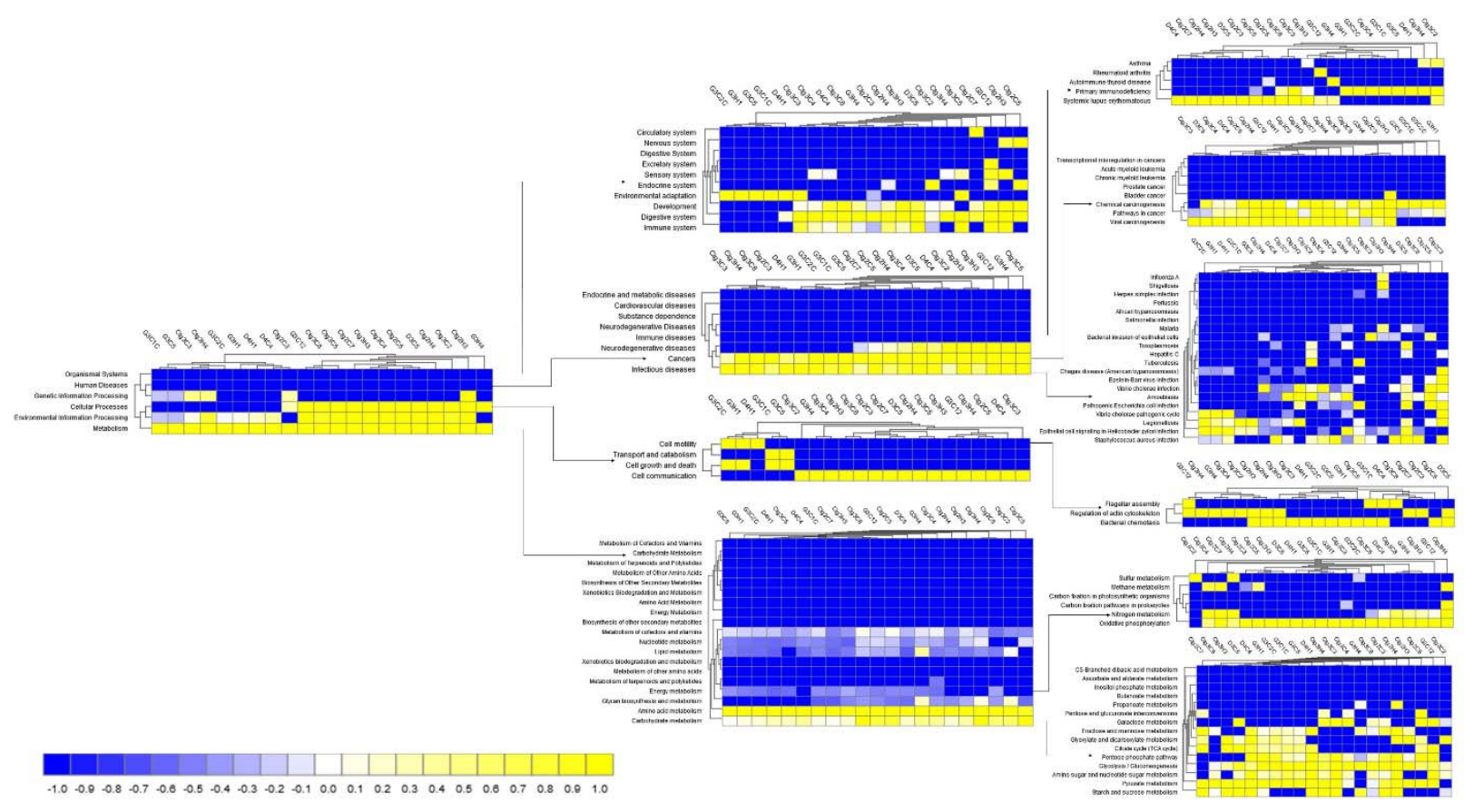

Fig. 6 Shotgun whole metagenome sequencing (WMS) reveals differences in functional microbial pathways. Heatmaps show the average relative abundance hierarchical clustering of the predicted KEGG Orthologs (KOs) functional pathways of the microbiota across all samples. The color bar at the bottom represents the relative abundance of putative genes. The color codes indicates the presence and completeness of each KEGG module, expressed as a value between -1 (low abundance), and 1 (high abundance). The greener colors indicates the more abundant patterns, whilst redder cells accounts for less abundant, and the dark black cells represents the absence of the KOs in that particular sample. Sample name: suffix ends with C refers to clinical mastitis $(\mathrm{CM})$ and that ends with $\mathrm{H}$ refers to healthy $(\mathrm{H})$ milk samples. 


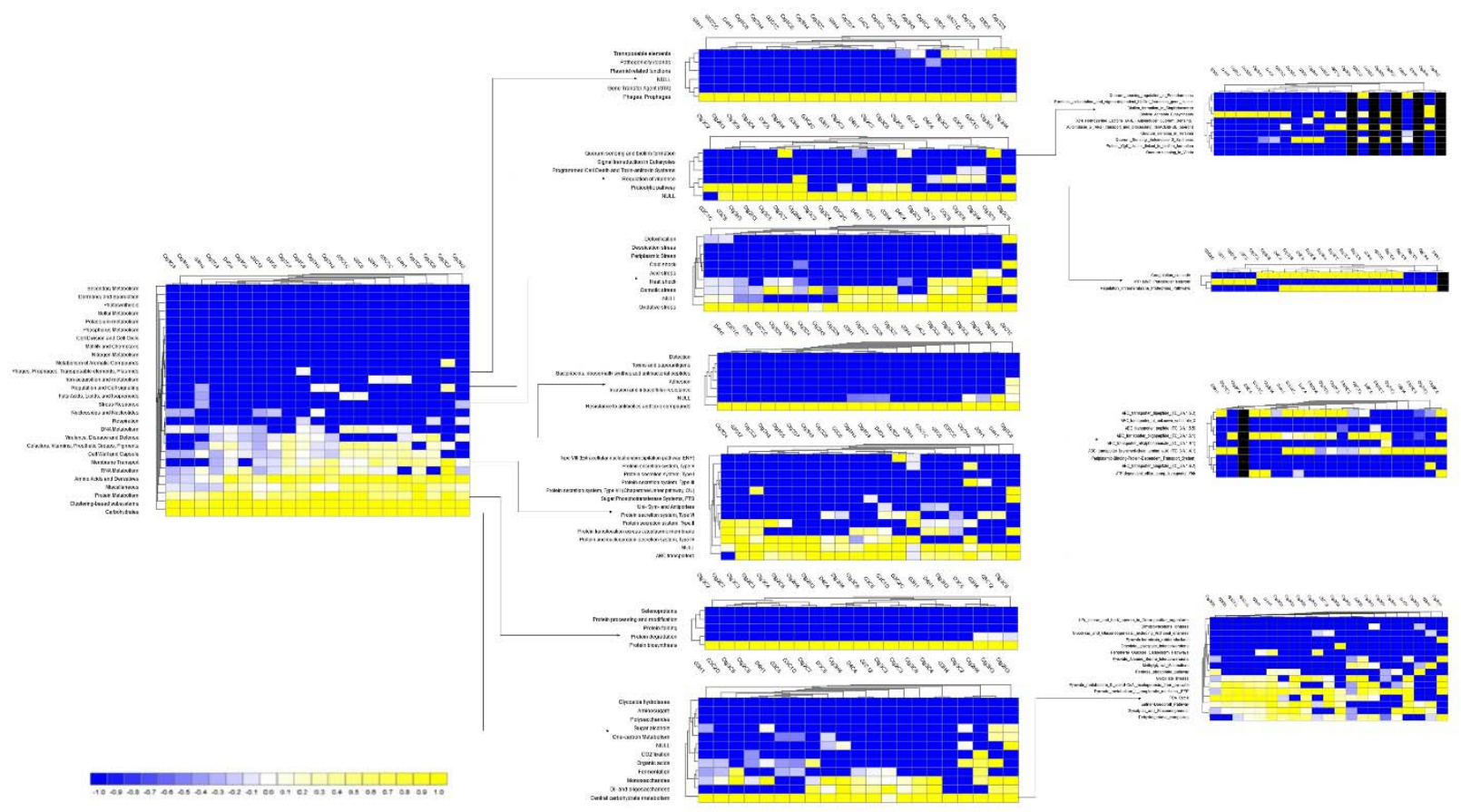

Fig. 7 Functional annotation using the SEED subsytem definition. Comparison of metagenomic profiles at the SEED subsystem level 3. Only a selection of subsystems showing significant differences between the two sample groups is shown. The subsystems less abundant in a given metagenome are shown in blue, and more abundant subsystems are represented in yellow colors. The color codes indicated the presence and completeness of each subsystem module, expressed as a value between -1 (low abundance), and 1 (high abundance). The color bar at the bottom represents the higher relative abundance of putative genes. Sample name: suffix ends with $\mathrm{C}$ refers to clinical mastitis $(\mathrm{CM})$ and that ends with $\mathrm{H}$ refers to healthy $(\mathrm{H})$ milk samples. 


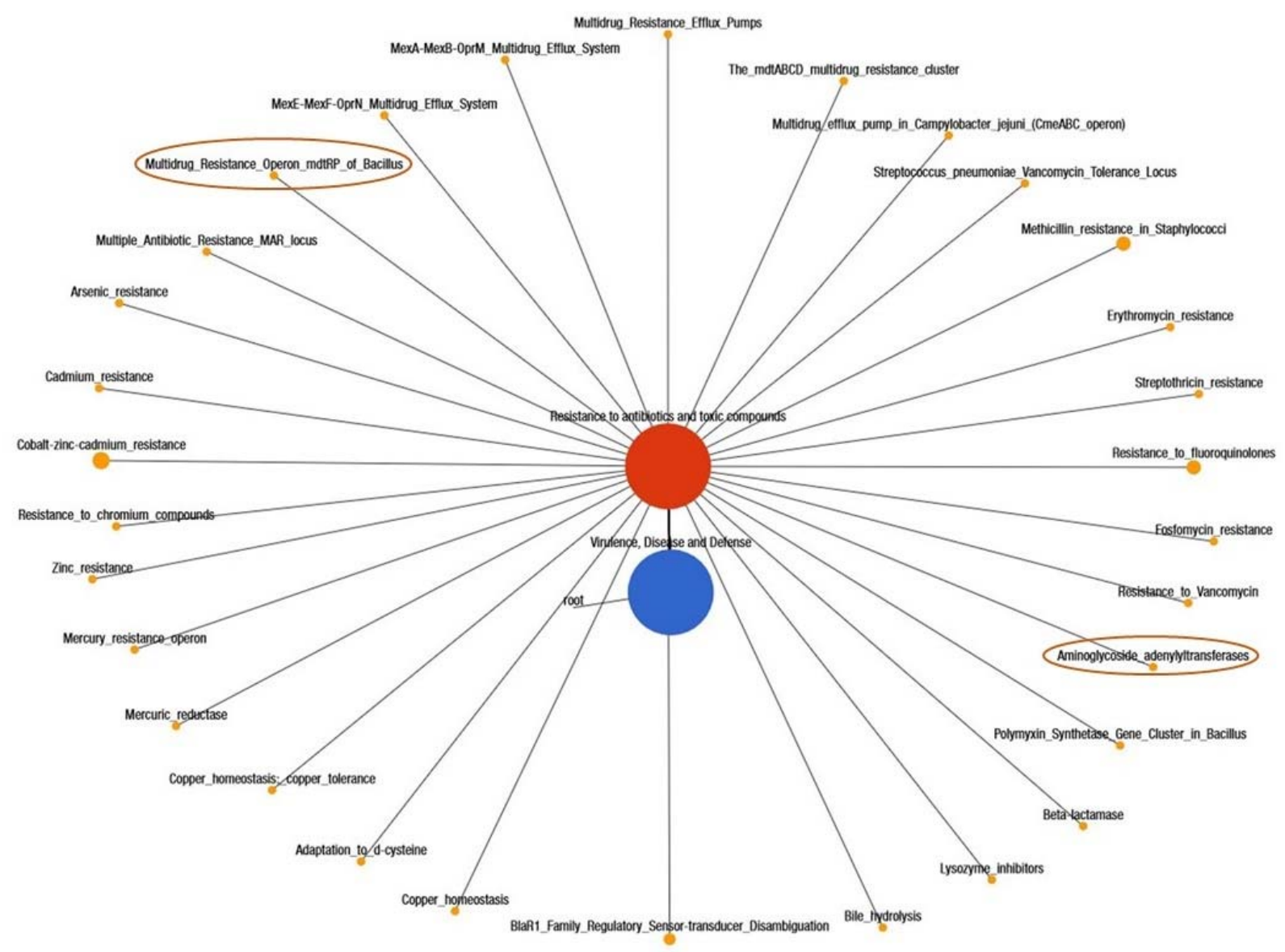

Fig. 8 Networks showing distribution of the antibiotics and toxic compounds resistance genes in clinical mastitis and healthy milk samples as detected in subsystem level 3 by using Euclidean distances in MG-RAST. A total of 30 genes in clinical mastitis and 28 in healthy milk metagenomes have been detected. Black lines with yellow circles demarcate the distribution of the resistant genes according to their class across the both metagenomes. The diameter of the circles indicates the relative abundance of the respective genes in both clinical mastitis and healthy milk samples. The two differentially expressed genes (multidrug resistance to operon, 
bioRxiv preprint doi: https://doi.org/10.1101/591982; this version posted April 7, 2019. The copyright holder for this preprint (which was not certified by peer review) is the author/funder. All rights reserved. No reuse allowed without permission.

$m d t \mathrm{RP}$ and aminoglycoside adenyltransferase) in clinical mastitis are highlighted in deep yellow circles. 